\title{
Metagenomic analysis of colorectal cancer datasets identifies cross-cohort microbial diagnostic signatures and a link with choline degradation
}

\section{Authors:}

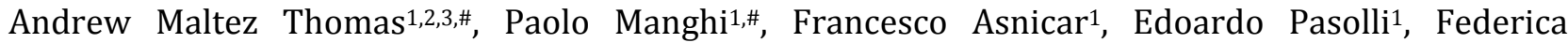
Armanini ${ }^{1}$, Moreno Zolfo ${ }^{1}$, Francesco Beghini ${ }^{1}$, Serena Manara ${ }^{1}$, Nicolai Karcher ${ }^{1}$, Chiara Pozzi ${ }^{4}$, Sara Gandini $^{4}$, Davide Serrano ${ }^{4}$, Sonia Tarallo ${ }^{5}$, Antonio Francavilla ${ }^{5}$, Gaetano Gallo6,7, Mario Trompetto ${ }^{7}$, Giulio Ferrero ${ }^{8}$, Sayaka Mizutani ${ }^{9,10}$, Hirotsugu Shiroma9, Satoshi Shiba11, Tatsuhiro Shibata11,12, Shinichi Yachida11,13, Takuji Yamada9 ${ }^{914}$, Jakob Wirbel15, Petra Schrotz-King16, Cornelia M. Ulrich ${ }^{17}$, Hermann Brenner16,18,19, Manimozhiyan Arumugam ${ }^{20,21}$, Peer Bork ${ }^{15,22,23,24}$, Georg Zeller ${ }^{15}$, Francesca Cordero $^{8}$, Emmanuel Dias-Neto ${ }^{3,25}$, João Carlos Setubal2,26, Adrian Tett ${ }^{1}$, Barbara Pardini' ${ }^{5,27}$, Maria Rescigno $^{28}$, Levi Waldron $29,30, *$, Alessio Naccarati5,31,*, Nicola Segata1,*,^

1 - Department CIBIO, University of Trento, Trento, Italy.

2 - Biochemistry Department, Chemistry Institute, University of São Paulo, São Paulo, Brazil.

3 - Medical Genomics Laboratory, CIPE/A.C. Camargo Cancer Center, São Paulo, Brazil.

4 - IEO, European Institute of Oncology IRCCS, Milan, Italy.

5 - Italian Institute for Genomic Medicine (IIGM), Turin, Italy.

6 - Department of Surgical and Medical Sciences, University of Catanzaro, Catanzaro, Italy.

7 - Department of Colorectal Surgery, Clinica S. Rita, Vercelli, Italy.

8 - Department of Computer Science, University of Turin, Turin, Italy

9 - School of Life Science and Technology, Tokyo Institute of Technology, Tokyo, Japan

10 - Research Fellow of Japan Society for the Promotion of Science

11 - Division of Cancer Genomics, National Cancer Center Research Institute, Tokyo, Japan

12 - Human Genome Center, The Institute of Medical Science, The University of Tokyo, Tokyo, Japan

13 - Department of Cancer Genome Informatics, Osaka University, Osaka, Japan

14 - PRESTO, Japan Science and Technology Agency, Saitama, Japan

15 - Structural and Computational Biology Unit, European Molecular Biology Laboratory, Heidelberg, Germany

16 - Division of Preventive Oncology, National Center for Tumor Diseases (NCT) and German Cancer Research Center (DKFZ), Heidelberg, Germany

17 - Huntsman Cancer Institute and Department of Population Health Sciences, University of Utah, Salt Lake City, Utah, USA

18 - Division of Clinical Epidemiology and Aging Research, German Cancer Research Center (DKFZ), Heidelberg, Germany

19 - German Cancer Consortium (DKTK), German Cancer Research Center (DKFZ), Heidelberg, Germany

20 - Novo Nordisk Foundation for Basic Metabolic Research, Faculty of Health and Medicine, University of Copenhagen, Denmark

21 - Faculty of Healthy Sciences, University of Southern Denmark, Odense, Denmark

22 - Molecular Medicine Partnership Unit (MMPU), Heidelberg, Germany

23 - Max Delbrück Centre for Molecular Medicine, Berlin, Germany

24 - Department of Bioinformatics, Biocenter, University of Würzburg, Würzburg, Germany

25 - Laboratory of Neurosciences (LIM-27), Institute of Psychiatry, University of São Paulo, São Paulo, Brazil.

26 - Biocomplexity Institute of Virginia Tech, Blacksburg VA 24061, USA

27 - Department of Medical Sciences, University of Turin, Turin, Italy.

28 - Mucosal immunology and microbiota Unit, Humanitas Research Hospital, Milan, Italy.

29 - Graduate School of Public Health and Health Policy, City University of New York, New York, USA.

30 - Institute for Implementation Science in Population Health, City University of New York, New York, USA.

31 - Department of Molecular Biology of Cancer, Institute of Experimental Medicine, Prague, Czech Republic.

\# These authors contributed equally

$49 *$ Co-senior authors

$50 \wedge$ Corresponding author nicola.segata@unitn.it 
Abstract

Several studies have investigated links between the gut microbiome and colorectal cancer (CRC), but questions remain about the replicability of biomarkers across cohorts and populations. We performed a meta-analysis of five publicly available datasets and two new cohorts, and validated the findings on two additional cohorts, considering in total 969 fecal metagenomes. Unlike microbiome shifts associated with gastrointestinal syndromes, the gut microbiome in CRC showed reproducibly higher richness than controls $(P<0.01)$, partially due to expansions of species typically from the oral cavity. Meta-analysis of the microbiome functional potential identified gluconeogenesis and the putrefaction and fermentation pathways to be associated with CRC, whereas the stachyose and starch degradation pathways were associated with controls. Predictive microbiome signatures for CRC trained on multiple datasets showed consistently high accuracy in datasets not considered for model training and independent validation cohorts (average AUC 0.84). Pooled analysis of raw metagenomes showed that the choline trimethylamine-lyase gene was over-abundant in CRC $(P=$ 0.001 ) identifying a novel relationship between microbiome choline metabolism and CRC. The combined analysis of heterogeneous CRC cohorts thus identified reproducible microbiome biomarkers and accurate disease-predictive models that can form the basis for clinical prognostic tests and hypothesis-driven mechanistic studies. 


\section{Introduction}

80 Colorectal cancer (CRC) is the second most common non sex-specific cancer and is responsible for more deaths than any other cancer after lung cancer ${ }^{1}$. Because of demographic trends toward an ageing population, the global incidence rate is expected to increase by nearly $80 \%$ to 2.2 million cases per year over the next two decades ${ }^{2}$. Sporadic CRCs, as opposed to hereditary CRCs, account for approximately $70 \%-87 \%$ of cases ${ }^{3}$ and genetics can only explain a small proportion of disease incidence ${ }^{4}$. The missing strong link of CRC with genetics points to the potential role of other variables including lifestyle and environmental factors as disease co-determinants. Reported risk factors associated with CRC include age, tobacco and alcohol consumption, lack of physical activity, increased body weight, and diet 5,6 . However, many non-genetic risk factors are common to several cancer types and these factors remain largely unsettled for CRC 7,8.

The human gut microbiome - defined as the microbial communities that populate our intestinal tract - is emerging as a relevant factor in human diseases 9,10 . Supported by some evidence of carcinogenic mechanisms induced by bacterial organisms $11-13$, the gut microbiome has also been hypothesized to play a crucial role in the development of CRC. Studies using 16S rRNA gene amplicon sequencing have led to the discovery of Fusobacterium nucleatum's association with CRC 14, which was subsequently shown to be causal in animal models of CRC carcinogenesis and progression ${ }^{15,16}$. Compared to $16 \mathrm{~S}$ rRNA gene studies, a smaller number of metagenomic sequencing studies have linked other microbial species and potential functional activities of the gut microbiome to CRC 17-19. However, the reproducibility and predictive accuracy of these high-resolution microbial signatures across cohorts and study design choices remain unclear. The potential use of the gut microbiome as a diagnostic tool for CRC has been proposed 17-21, but not yet validated across multiple independent study populations.

There is thus a need to establish and validate links between the human gut microbiome and CRC carcinogenesis across populations, cohorts, and microbiome tools. Some multi-cohort works have been performed based on $16 \mathrm{~S}$ rRNA gene studies ${ }^{22}$, but this technique has important technical limitations ${ }^{23}$. The recent availability of whole-metagenome shotgun datasets of CRC cohorts ${ }^{17-21}$ enables a combined multi-population exploration of the CRC-associated microbiome with strain-level resolution ${ }^{24,25}$ and meta-analytic predictive approaches ${ }^{10,26}$, but the only meta-analysis study performed so far on CRC is affected by overfitting issues ${ }^{27}$. It is thus crucial to perform large-scale cross-cohort studies to provide an unbiased and well-powered assessment of the link between CRC and the gut microbiome.

In this study, we have sequenced 140 samples from two different cohorts, performed an integrated analysis combining all current metagenomic CRC datasets available, and assessed prediction accuracies of the gut microbiome for CRC detection across populations, datasets, and conditions.

\section{A meta-analysis of metagenomic datasets to identify links between the gut microbiome and} 115 CRC

116 To identify reproducible relationships between the gut microbiome and CRC, we performed shotgun metagenomic sequencing ${ }^{28}$ of the stool microbiome of 140 CRC patients and controls recruited in two cohorts, and analyzed these in the context of 624 additional samples from five publicly available and geographically diverse metagenomic studies. We validated the results on two novel datasets of $60 \mathrm{CRC}$ and 65 controls ${ }^{29}$ and $40 \mathrm{CRC}$ and 40 controls (see Methods), respectively. In total, we 
considered 413 samples from CRC patients, 143 from subjects with adenoma and 413 control samples. Participants from all studies underwent colonoscopy to diagnose CRC, adenoma, or to confirm the absence of disease, with samples collected before diagnosis or beginning of treatment (Suppl. Table 1, Table 1). All datasets were sequenced at high depth except for the Hannigan et al. study ${ }^{30}$ (Extended Data 1A, Methods).

\section{Meta-analysis shows higher species richness in CRC-associated samples}

We first tested whether microbial richness and diversity differed between CRC samples and controls, given contrasting current evidence ${ }^{31-33}$. In all but one study, the median species richness was higher in CRC samples compared to controls, and the increase was significant in four of the six deeply sequenced datasets $(P<0.05$ Extended Data 1B-C). Meta-analysis of standardized mean differences by random effects model for the number of microbial species confirmed the higher number of species in CRC compared to controls $(\mu=0.5,95 \% \mathrm{CI}[0.16,0.85], P=0.004)$, although with significant heterogeneity across datasets $\left(\mathrm{I}^{2}=74.8 \%, \mathrm{p}=0.0007\right.$, Q-test $)$. This difference was not meaningfully affected when controlling for potential confounding by age, BMI, or sex(Extended Data 1D-E). Conversely, we observed no difference in diversity between carcinomas and controls (Extended Data 2A-B). We thus provide strong evidence that the CRC-associated microbiome has a quantitative species distribution which is consistent with healthy controls, but is significantly enriched in the total number of detected microbes.

We further tested whether the CRC-associated microbiome possesses more oral cavity-associated species than controls, as previously hypothesized ${ }^{22,34}$. Considering the 161 species we identified from multiple existing datasets 35,36 as being typical colonizers of the oral cavity (see Methods), we found increased oral species richness in CRC samples for all but one of the six deeply sequenced datasets compared to controls and the increase was significant in meta-analysis $(\mu=0.16,95 \%$ CI $[-0.03,0.35]$, $P=0.02$, Extended Data 2G). Similarly, the total abundance of oral species in the stool microbiome was also significantly higher in CRC patients compared to controls (meta-analysis $\mu=0.23,95 \%$ CI $[0.07,0.39], P=0.003)$. Altogether, greater species richness and abundance may be a sign of an altered gut microbiome in CRC, and it is indicative of an influx of bacterial species originating from the oral cavity.

\section{A panel of microbial biomarkers for CRC is reproducible across cohorts}

Individual biomarker discovery efforts can be sensitive to technical artefacts and to heterogeneity of factors implicated in microbial shifts in healthy populations, including biogeography, diet, and host genetics 25,37 . This is confirmed by the two newly sequenced datasets that have only partially overlapping taxonomic and functional potential biomarkers (Extended Data 3). Even so, several CRC biomarker species were identified by univariate statistics 38 independently in the majority of the datasets: F. nucleatum, Solobacterium moorei, Porphyromonas asaccharolytica, Parvimonas micra, Peptostreptococcus stomatis, and Parvimonas ssp. Other species were identified in fewer datasets or were dataset-specific (Figure 1A, and Suppl. Table 2). F. nucleatum, whose connection with CRC has been extensively reported 14,17-19, had significantly increased abundance in CRC patients in all datasets with adequate sequencing depth, when considering single markers for this species (Extended Data 4A). Some of the cross-cohort CRC biomarker species have already been reported $14,22,34$ and many of them are commonly found in the oral cavity (8 out of the 39 total biomarkers found in at least 2 datasets), consistent with the increased oral taxa presence in CRC samples mentioned above. 
We then pooled evidence of differential abundance across datasets by random effects meta-analysis. Among the 26 differentially abundant species at FDR $<0.005$, those with the highest effect size were again F. nucleatum, S. moorei, $P$. asaccharolytica, $P$. micra and $P$. stomatis. The meta-analysis additionally identified Clostridium symbiosum, which has been tested as a marker for early CRC detection 39 (Figure 1B). Other differentially abundant species at FDR $<0.05$ have not been previously reported in CRC microbiome studies, including Streptococcus tigurinus and Streptococcus dysgalactiae, and 3 different Campylobacter species. We also confirmed Gemella morbillorum and Streptococcus gallolyticus to be relevant biomarkers, as previously suggested in smaller cohorts 18,40 . In contrast, only 12 species were associated with the control population in the meta-analysis and only four were significantly enriched for the same populations in at least three datasets. Controlassociated species with the highest effect sizes were Gordonibacter pamelae and Bifidobacterium catenulatum (Figure 1B, Suppl. Table 2; Extended Data 4C), which are generally considered beneficial microbes and have been used as probiotic supplements ${ }^{41}$. Adjustment for potential confounding by host characteristics did not meaningfully affect crude estimates in the meta-analysis (Figure 1D, Extended Data 4B). The substantially higher number of species enriched in CRC than in controls ( 49 vs. 12), even when focusing only on species with putative oral origin (15 vs. 2, Extended Data 5A), points to the existence of a reproducible taxonomic signature of the CRC-associated microbiome.

Functional potential of the microbiome was also significantly associated with CRC samples when compared against healthy controls. We found overall increased richness of UniRef gene families 42 in CRC samples in two datasets, with percentages of unmapped reads ranging between $20 \%$ and $40 \%$ (Extended Data 5E). We found 33,840 of the 2,479,274 single gene families detected at least once to be associated with CRC and 30,475 associated with controls (FDR $<0.05,9,154$ and 7,115 differential gene families at FDR $<0.005$ respectively). We further observed 136 out of 590 metagenomically reconstructed microbial functional pathways to be CRC-associated, and only 37 associated with controls (Suppl. Table 3). Among the most differentially abundant pathways (Figure 1C) that are at worst just minimally affected by potential confounding factors (Figure 1E), we found starch, stachyose, and galactose degradation to be associated with controls. These associations could indicate how potentially diet-associated changes in the functional repertoire of the microbiome can influence host conditions. The CRC-associated microbiome showed an association with gluconeogenesis and with capacity for uptake and metabolism of amino acids via putrefaction and fermentation pathways (Suppl. Table 3-4). These included those pathways responsible for the conversion of different amino acids to tumor-promoting compounds 19,43 , such as polyamines (e.g. Larginine and L-ornithine degradation to putrescine) and ammonia (L-histidine and L-arginine degradation, and L-lysine and L-alanine fermentation to acetate, butyrate and propionate). These pathways (Figure 1C) and the set of species described above (Figure 1A,B) thus constitute a collection of microbiome biomarkers that is reproducible across cohorts.

\section{Predicting CRC from single metagenomic datasets in independent cohorts leads to reduced accuracy}

To test the hypothesis that the stool microbiome could be used as a reproducible CRC pre-screening tool, we performed intra-cohort, cross-cohort and combined-cohort prediction validation on the overall set of 621 CRC and controls samples using a Random Forest classifier (Table 1). In intracohort cross-validation using species-level taxonomic relative abundances, we observed performances ranging from 0.92 to 0.58 AUC score, with an average in the deeply sequenced datasets of 0.81 AUC (Figure 2A). When using the functional potential of the gut microbiome by means of 
pathway abundances, we observed decreased single dataset cross-validation accuracies, with the exception of our Cohort1 (maximum 0.82 AUC, average 0.71 AUC, Extended Data 6A). The profiling of the more fine-grained UniRef90 gene family abundances improved the predictions, with AUCs reaching 0.84 AUC for Cohort2 and an average of 0.77 AUC in the deeply sequenced datasets (Figure 2B). These results show that, while cross validation AUCs can be high for predicting CRC in some datasets, they are highly variable and dataset dependent.

We then tested whether and how much the microbial signatures of CRC remained predictive across distinct datasets and cohorts. To this end, we trained the classifier on each single "training" dataset and applied the model on each distinct "testing" dataset. For most datasets this led to decreased AUC values when compared to single cross validation AUCs, and AUCs showed a high variability across cohorts (minimum 0.5 and maximum 0.86 cross dataset AUC). These results were consistent when using either pathway or gene family-abundances as predictors (Extended Data 6and Figure 2B). Overall, we highlight a poor transportability of the microbiome signature from one dataset to the other and experimental choices ${ }^{44}$ and cohort or population characteristics ${ }^{25}$, may explain the reduced cross-study predictability when considering single datasets to train the model (Extended Data 6C-D).

\section{Pooling of training cohorts substantially improves prediction across datasets}

To overcome the limitations of training on single datasets (Suppl. Table 5), we performed a LeaveOne-Dataset-Out (LODO) analysis 45 in which classifiers were trained on six datasets combined, and validated on the left-out dataset, for each dataset in turn. For taxonomic profiles, this approach improved both AUC values and inter-dataset consistency, producing AUCs $\geq 0.80$ (average 0.84 s.d. 0.03 ) for all six deeply sequenced datasets (Figure 2A). Predictors based on clade-specific markers also produced high, albeit more variable AUC values, outperforming taxonomic profiles in some datasets (Extended Data 6B). Gene families achieved slightly reduced performances, whereas pathway abundances produced substantially less accurate predictions (Figure 2B). The technical and host population diversity embedded in these training meta-cohorts may be crucial in improving the generalizability of classifiers, as we found this LODO approach to be substantially and consistently more informative than a single-dataset cross-validation, and independent investigations found similarly high LODO performances using different metagenomic profiles and machine learning tools 29.

The model trained on taxonomic or functional features was also shown to capture the above wholemicrobiome biomarkers because the direct inclusion of alpha-diversity metrics, oral-species abundance, and a measure of metagenome mappability did not provide substantial improvements (mean 0.83 , s.d. 0.03 for the deeply sequenced datasets when using the taxonomic model). However, based on the performance and variability of the predictive models across datasets, we recommend using species-level microbial abundance as the main feature set for CRC status prediction in a LODO setting.

To assess the relation between population diversity in the training meta-cohort and prediction performance, we considered increasingly larger subsets of the available training cohorts. AUC values sharply increased when moving from one to two training datasets (10\% to $13 \%$ median AUC improvement depending on the features considered in the model, Extended Data 7) with less marked improvements at further dataset additions (Figure 2C-D). Large and heterogeneous combined training sets thus generate improved accuracy for identifying CRC cases in independent metagenomic datasets. 
255 The predictive CRC-associated microbiome signatures identified above considered all observed 256 species and gene functions and would thus be impractical for clinical application without whole microbiome profiling. We thus sought to identify a minimal set of highly predictive microbial features by exploiting the internal feature ranking of the Random Forest classifier ${ }^{10}$. We found that $P$. stomatis was the species with the highest average rank. As expected, other CRC-associated species such as $F$. nucleatum, Parvimonas ssp., P. asaccharolytica, G. morbillorum, Clostridium symbiosum and P. micra were also crucial to prediction accuracy (Figure 3A) with the top seven ranked species for CRC detection amongst those with the largest effect sizes in the meta-analysis. Very few species were ranked high in the learning models, further highlighting that successful discrimination is achieved by 264 CRC-specific rather than control-specific microbial features.

To evaluate how many microbial species or gene families are necessary to achieve prediction scores comparable to those obtained using the full set of features, we computed AUC values at increasing numbers of features. Feature ranking was performed internally to each training fold to avoid overfitting. By applying this approach to all datasets (Figure 3B-C), we found that using as few as 16 species achieved CV AUC $>0.8$ for the majority of the datasets, with little improvement from using all remaining species ( $2 \%$ improvement in the mean AUC value). We also found that using only 64 gene families achieved prediction values $>0.8$ for the same datasets, and that using all 8,192 gene families improved AUC only slightly (2\% improvement -Extended Data 8). Therefore, these results suggest that a stool-based diagnostic test using genetic markers targeting a limited number of microbial species or genes would serve as a promising clinical tool.

\section{Microbiome signatures for adenomas are only partially predictive}

We assessed the ability to discriminate adenomas from controls or carcinomas, using 27 newly sequenced adenoma-associated samples and 116 adenoma-associated samples from available studies (Table 1). Adenomas could be distinguished from CRC patients with lower accuracy than controls (mean AUC 0.69 versus 0.79, Extended Data 6E-F) and there are only eight species that differentiate adenoma patients from carcinoma patients in the meta-analysis (FDR $<0.05)$. Seven of these eight biomarkers are in common with the comparison between carcinoma patients and healthy individuals, and the LODO approach did not improve discrimination of adenomas from CRC (average AUC 0.68). Moreover, we found that no dataset could accurately predict adenomas from control samples (maximum AUC 0.58, minimum 0.46), even when using a LODO approach (average AUC 0.54). In the meta-analysis, no species were significantly different when contrasting samples from patients with adenomas and healthy controls. These results reinforce previous findings 18,19 that the adenomaassociated stool microbiome closely resembles that of the healthy gut.

\section{Increased abundance of choline TMA-lyase encoding genes in CRC}

Microbiome-derived metabolites and specifically polyamines have been implicated in carcinogenesis both in animal models and in humans ${ }^{43}$. We chose to focus on trimethylamine (TMA), an amine produced by bacteria from choline and carnitine, because it has been shown to play a role in complex diseases such as atherosclerosis and primary sclerosing cholangitis 9,46. Since dietary components have been linked with CRC risk 5,6, we hypothesized that the TMA-producing potential of the human gut microbiome could also be associated to CRC ${ }^{47}$. To test this hypothesis, we considered the genes belonging to the main TMA-synthesis pathways to reconstruct and quantify the presence of such genes in the CRC-associated metagenomes. The main genes associated with TMA-synthesis are those encoding the choline TMA-lyase (cutC), the L-carnitine dioxygenase (yeaW) and the L- 
carnitine/gamma-butyrobetaine antiporter (caiT) and we identified them in 923, 5,185 and 5,709 available bacterial genomes, respectively.

Screening the 7 CRC-associated metagenomic datasets, we found that only one of them had a significant increase of caiT in CRC samples compared to controls, whereas no significant differences were detected for yeaW (Extended Data 9A). However, we found increased abundance of cutC in CRC samples compared to controls in all seven datasets $(P<0.05$ by Wilcoxon Rank Sum test on RPKM abundances for five datasets, Figure 4A). Meta-analysis indicated an overall strong association with no evidence of heterogeneity $\left(P=0.001, \mu=0.27,95 \% \mathrm{CI}[0.1,0.42], \mathrm{I}^{2}=4.2 \%\right.$, Q-test $=0.65$, Figure 4B). We also analyzed the abundance of the gene encoding the choline TMA-lyase-activating enzyme (cutD), finding a significant increase in CRC (meta analysis $P=0.001, \mu=0.32,95 \%$ CI [0.16, 0.47], $\mathrm{I}^{2}=0 \%$, Q-test $=0.96$, Extended Data 9B-C). These results indicate that TMA production might happen preferentially via choline degradation, and not via carnitine, and could substantially affect the amounts of TMA and trimethylamine oxide (TMAO) in an individual ${ }^{48}$. Intermediate levels of cutC in adenomas (Figure 4A) is further suggestive of a TMA action along the adenoma-carcinoma axis. We validated the increased cutC gene abundance in CRC by qPCR ${ }^{49}$ on a subset of samples from Cohort1 with enough DNA left after sequencing, and confirmed the metagenomic findings (one-tailed Wilcoxon signed rank test $P=0.024$, Figure 4D). Further quantification of $c u t C$ transcript abundance from the co-extracted RNA in the same dataset also pointed to an over-expression of this gene in CRC $(P=0.035$, Figure 4E).

We further explored the role of cutC in the gut microbiome by reconstructing sample-specific sequence variants using a reference-aided targeted assembly approach (see Methods). We found a large sequence divergence for the gene encoding this enzyme that is known to occur in single copies in the genomes ${ }^{49}$ and we identified four main sequence variants that are associated with the taxonomic structure (Figure 4B, Extended Data 9C-D, 10A-B). Interestingly, the most prevalent $(46.5 \%)$ cutC sequence type belonged ( $>95 \%$ identity over the full lenght of the gene) to an unknown species that was only recently assembled from metagenomics 50 and assigned to species-level genome bin (SGB) ID 3957. This candidate species comprises 56 metagenomically-assembled species 50 and is placed within the Lachnospiraceae family, but the missing genus assignment confirms that several microbes remain under-characterized in the human microbiome. This cutC variant was associated with non-CRC samples (OR 0.38, 95\% CI [0.25, 0.57], $P=0.0001$, Fisher Test), whereas cutC sequence types mostly belonging to Hungatella hathewayi and Clostridium asparagiforme (Firmicutes) were significantly CRC-associated (OR 2.14, 95\% CI [1.29, 3.56], $P=0.004$, Fisher test), as were sequence types belonging to Klebsiella oxytoca and Escherichia coli (OR 1.85, 95\% CI [1.13, 3], $P=0.02$, Fisher Test - Figure 4B). Altogether, these novel findings highlight that sequence variants of cutC can be strongly associated with disease, potentially because of corresponding differences in the efficacy of choline degradation and TMA production.

\section{Additional independent validation of predictive models}

To further validate our meta-analysis results, we considered two additional independent metagenomic cohorts from Germany ${ }^{29}$ (Validation Cohort1) and Japan (Validation Cohort2) comprising a total of $100 \mathrm{CRC}$ patients and 105 controls (see Methods). The metagenomic predictive model was confirmed to be highly accurate on these new cohorts (Figure 5A) with an AUC of 0.90 and 0.81 for the German and Japanese cohorts respectively, when using the species-level taxonomic abundance model. Species newly associated to the CRC microbiome such as Streptococcus tigurinus and Streptococcus dysgalactiae were confirmed to have higher prevalence in CRC than in controls In the two validation datasets (blocked Wilcoxon test ${ }^{51} P=0.049$ and $P=0.011$ for $S$. tigurinus and $S$. 
dysgalactiae, respectively). Enrichment in the CRC-associated microbiome of these two species was confirmed also by the analysis of additional metagenomic datasets of IBD 52 and type-2 diabetes 53,54 in which the prevalence of $S$. tigurinus was always below $10 \%$ in both cases and controls, whereas $S$. dysgalactiae was never detected in these additional datasets. We also confirmed species richness to be significantly higher in CRC $(P=0.0005$ for both validation datasets after rarefaction at the 10th percentile, Figure 5B) as well as richness of oral microbial species in the rarefied samples (blocked Wilcoxon test $\left.{ }^{51} P=0.003\right)$, and the abundance of the gene encoding the choline TMA-lyase enzyme cutC in CRC $(P<1 \mathrm{e}-6)$.

\section{CRC-specificity of microbiome predictive models}

We performed additional experiments to validate the discriminative power of the above microbial signatures specifically for CRC and not for other potentially microbiome-linked disease conditions. To this end, we first considered 13 additional fecal samples sequenced from patients that underwent colonoscopy in our Cohort 1 that were originally discarded because the final diagnosis pointed at diseases other than adenomas or carcinomas such as ulcerative colitis, Crohn's disease, uncategorized colitis, and diverticular diseases. These were distinguishable from CRC samples based on the taxonomic model ( 0.78 cross-validation AUC, 0.80 AUC using only 16 species), and only slightly decreased the AUC of the model trained on all the other datasets when they were added to the non-disease (i.e. healthy) category (from 0.83 to 0.79 in AUC). We then expanded this analysis to diseases for which at least two distinct large metagenomic datasets are available in the public domain and this includes ulcerative colitis (UC) and Crohn's disease (CD) ${ }^{52,55}$ as well as non-GI diseases such as type-2 diabetes ${ }^{53,54}$. For this purpose we added samples randomly drawn from each of the case and control conditions of these additional disease cohorts to the control class of the new validation cohort and recorded the variations in AUCs when attempting to predict CRC (see Methods). By comparing the AUCs obtained when adding non-CRC external cases and when adding the corresponding external controls, we found for both validation cohorts a small decrease in prediction accuracy for both UC (3\% and 4\% for Validation Cohort1 and Validation Cohort2, respectively; Figure 5C) and $\mathrm{CD}$ (5\% and 9\%, for Validation Cohort1 and Validation Cohort2, Figure 5C), pointing to a limited effect on the CRC model of samples from these two diseases. For type-2 diabetes we observed an increase in the predictive power in one dataset ${ }^{53}$, and a decrease in the other ${ }^{54} \mathrm{in}$ both validation datasets, and the CRC model always remained highly predictive (AUC $\geq 0.80$ ). Altogether, these results point at the existence of a clear microbiome signature of CRC which is distinct from other relevant diseases with a gastrointestinal component.

\section{Relationship to currently available non-invasive clinical screening tests}

To assess the potential of microbiome-based prediction models in comparison and in combination with currently used non-invasive clinical screening tests, we considered the Fecal Occult Blood Test (FOBT) and the Wif-1 Methylation test available for 110 samples of the ZellerG_2014 cohort ${ }^{19}$. The LODO microbiome model tested on this dataset proved to be slightly superior to the FOBT at multiple combinations of specificity and sensitivity levels (Figure 5D) and on par with the Wif-1 Methylation test. Considering the LODO model predictions and the FOBT together in the same test improves the sensitivity/specificity trade-off at high specificity levels when the integration is based on having at least one predictor positive, and at relatively lower specificity levels when requiring both predictors to be positive (Figure 5D). Integrating the microbiome model with the Wif-1 Methylation test results in similar performances, and the use of the reduced microbiome model with only 16 species generally improves the results (Figure 5D). We thus provide evidence for the potential clinical value of 
microbiome predictive models especially when considered together with other available noninvasive clinical tests.

\section{invasive clinical tests.}

\section{Discussion}

In the present study, we comprehensively assessed the CRC-associated gut microbiome and its ability to distinguish newly diagnosed CRC patients from tumor-free controls. Our study was performed across multiple datasets and populations, through a combined analysis of fecal CRC metagenomes from four previously unpublished cohorts and five publicly available datasets. Whereas direct specific host-microbe interactions have been shown to cause certain malignancies in vitro and in vivo animal models $11-13,56$ and genotoxic determinants such as colibactin tend to be over-represented in the analyzed datasets 29 , indirect metabolite-mediated mechanisms may be more important to the development of carcinomas although causality relations need to be tested. In our analysis, we indeed found a reproducible panel of microbiome species (Figure 1), whole microbiome characteristics, and strain-level biomarkers (Figure 4) beyond the validated mechanisms of specific variants of Escherichia coli 11,56 and Bacteroides fragilis ${ }^{56}$. We found that the gut microbiome in CRC has greater richness than controls, partially due to the presence of oral cavity-associated species rarely found in healthy guts, challenging the widespread assumption that decreased alpha-diversity is generally associated with intestinal dysbiosis 57,58 .

The identification of reproducible microbial biomarkers for CRC may enable the design of noninvasive diagnostic tools. We developed machine learning models able to distinguish between carcinoma patients and controls with an average performance above 0.84 AUC when validated on datasets excluded from the training of the model (Figure 2A). Importantly, these performances are quite independent of specific methodological choices given that complementary investigations 29 using different metagenomic profilers and machine learning approaches achieved very similar results. Further increase in prediction performance can be achieved using larger datasets $(n>1,000)$ rather than different methodologies (Figure 2C-D, Figure 5C), and the combination of a microbiome model with other clinical tests and patient risk factors could substantially improve this diagnostic accuracy (Figure 5D). Current clinical pre-colonoscopy screening tests (e.g. FOBT, WIF-1) remain cheaper, but the microbiome-based CRC prediction models enable a very high diagnostic potential which increases with the number of microbes or microbial genes used, with single biomarkers being much inferior to multi-featured diagnostic models. However, nearly maximal accuracy was achieved with as few as 15 to 25 microbes (Figure 3B-C) or a few hundred genes (Extended Data 8), potentially enabling inexpensive clinical microbiological tests to be performed on stool. Prospective studies of these biomarkers are needed to establish whether they can identify individuals at elevated risk of CRC and provide the possibility of disease prevention.

The diversity and subject-specificity of the human gut microbiome is not yet fully uncovered, with many microbial genes having unknown function, and with strain-level diversity that is missed by many current analysis pipelines ${ }^{50}$. Large scale shotgun metagenomics can begin to overcome these limitations, as shown here by the novel identification of a link between CRC and the microbial pathway producing trimethylamine from choline ${ }^{48}$. The gene encoding for the key enzyme for this pathway, the CutC choline TMA-lyase, is both more overall abundant and expressed in the gut microbiomes of carcinoma patients, with specific variants of cutC characterizing controls, adenomas, and carcinomas (Figure 4). TMA-producing choline lyases have been found to be associated with atherosclerosis ${ }^{9}$, and higher plasma trimethylamine oxide and choline levels have been reported to 
be correlated with CRC risk ${ }^{59,60}$. We highlighted the importance of strain-level gene resolution in understanding any potential carcinogenic role of cutC. CRC-associated variants mostly originated from Hungatella hathewayi, Clostridium asparagiforme, Klebsiella oxytoca, and Escherichia coli, whereas no significant enrichment was detected for a cutC variant carried by a unexplored recently discovered candidate species in the Lachnospiraceae family ${ }^{50}$. Thus, genetic variants in key microbial genes involved in choline-induced TMA production by the gut microbiome are a plausible and novel potential mechanism for colorectal carcinogenesis. Other partially diet-dependent microbiome factors can contribute to promote carcinogenesis, and we found in our parallel work that genes for secondary bile acid conversion are consistently enriched in the CRC-associated microbiomes ${ }^{29}$. Further work is needed to establish the changes in protein structure and function associated with the genetic variants of the diet-related microbial genes found here to be enriched in the CRC microbiome.

Analysis of cancer cohorts that are heterogeneous for geography, ethnicity, and lifestyle, presents a distinct opportunity for studying the cancer-associated microbiome. By combining multiple small cohorts of potentially low generalizability, it is possible to obtain better representation of the spectrum of cancer cases and controls. With appropriate methodology, artifactual findings due to batch effects present in any individual dataset can be avoided. The use of large, diverse training sets enables creation of more accurate diagnostic models, and the availability of independent validation datasets enables more realistic estimation of that accuracy. Future shotgun metagenomic studies of the intestinal mucosa-associated microbiome, which are currently infeasible due to excessive human DNA contamination 28 , will be important to further refine the list of CRC-associated gut microbes. Nevertheless, this study identifies highly reproducible microbial CRC biomarkers and points to the potential for non-invasive microbial diagnostic tests to supplement existing screening.

\section{Acknowledgements}

We thank the members of the Segata, Naccarati, and Waldron groups for insightful discussions, all the volunteers enrolled in the study, the NGS facility at University of Trento for performing the metagenomic sequencing, and the HPC facility at University of Trento for supporting the computational experiments. This work was primarily supported by Lega Italiana per La Lotta contro $\mathrm{i}$ Tumori to N.S., F.C. and A.N and by Fundação de Amparo à Pesquisa do Estado de São Paulo (FAPESP 16/23527-2) to A.M.T. This work was also partially supported by the Conselho Nacional de Pesquisa e Desenvolvimento (CNPq, Brazil) to J.C.S. and E.D.-N., FAPESP (14/26897-0), Associação Beneficente Alzira Denise Hertzog Silva (ABADHS, Brazil) and PRONON/SIPAR to E.D.-N., by Coordenação de Aperfeiçoamento de Pessoal de Nível Superior - Brazil (CAPES) - Finance Code 001 to J.C.S., by the Italian Institute for Genomic Medicine (IIGM) and Compagnia di San Paolo Torino to A.N, A.F., B.P. and S.T., by Fondazione Umberto Veronesi “Post-doctoral fellowship Year 2014, 2015, 2016, 2017 and 2018" to B.P. and S.T., by the Grant Agency of the Czech Republic (17-16857S) to A.N., by Fondazione Umberto Veronesi (FUV-14-SG-GANDINI) to S.G., by the European Union H2020 Marie-Curie grant (707345) to E.P., by the European Research Council (ERC-STG project MetaPG) to N.S., by MIUR "Futuro in Ricerca" RBFR13EWWI_001 to N.S., by the People Programme (Marie Curie Actions) of the European Union FP7 and H2020 to N.S., and by the National Cancer Institute (U24CA180996) and National Institute of Allergy and Infectious Diseases (1R21AI121784-01) of the National Institutes of Health to L.W. B.P. is recipient of a Fulbright Research Scholarships (year 2018). We acknowledge funding from EMBL, DKFZ, the Huntsman Cancer Foundation, the Intramural Research Program of the National Cancer Institute, ETH Zürich, and the following external sources: the European Research 
Council (CancerBiome ERC-2010-AdG_20100317 to P.B., Microbios ERC-AdG-669830 tp P.B.), the Novo Nordisk Foundation (grant NNF10CC1016515 to M.A.), the Danish Diabetes Academy supported by the Novo Nordisk Foundation and TARGET research initiative (Danish Strategic Research Council [0603-00484B] to M.A.), the Matthias-Lackas Foundation (to C.M.U.), the National Cancer Institute (grants R01 CA189184, R01 CA207371, U01 CA206110, P30 CA042014 ll to C.M.U.), the BMBF (the de.NBI network \#031A537B to P.B. and the ERA-NET TRANSCAN project 01KT1503 to C.M.U.), and the Helmut Horten Foundation (to S.Sunagawa). For the Validation Cohort2, funding was provided by grants from the National Cancer Center Research and Development Fund (25-A-4,28-A-4, and 29-A-6), Practical Research Project for Rare/Intractable Diseases from the Japan Agency for Medical Research and Development (AMED) (JP18ek0109187), JST (Japan Science and Technology Agency)-PRESTO (JPMJPR1507), JSPS (Japan Society for the Promotion of Science) KAKENHI (16J10135, 142558 and 221S0002), Joint Research Project of the Institute of Medical Science, the University of Tokyo, and the Takeda Science Foundation and Suzuken Memorial Foundation.

\section{Author contributions}

N.S., A.M.T., L.W., and A.N conceived the study. N.S. supervised the study. C.P., S.G., D.S., S.T., A.F., G.G., M.T., B.P, M.R., and A.N. organized the clinical study, recruited patients and collected samples. F.Armanini generated metagenomic data. A.M.T., P.M., F.Asnicar, E.P., M.Z., F.B., N.K., and G.F. collected and analyzed the metagenomic data. A.M.T., P.M., F.Asnicar, E.P., M.Z., G.F., J.W., G.Z., and L.W. performed machine learning and statistical analyses. F.Armanini, S.T., S.Manara, A.T., B.P, and A.N. performed validation experiments. S.Mizutani., H.S., S.Shiba, T.S., S.Y., T.Y., J.W., P.S.-K, C.M.U., H.B., M.A., P.B., and G.Z. provided additional validation data. A.M.T., P.M., L.W., and N.S. designed and produced the figures. A.M.T., P.M., and N.S. wrote the manuscript with contributions from S.Manara, F.C., E.D.-N., J.C.S., M.R., L.W., and A.N. All authors discussed and approved the manuscript.

\section{Competing Interests}

P. Bork, G. Zeller, A.Y. Voigt, and S. Sunagawa are named inventors on a patent (EP2955232A1: Method for diagnosing colorectal cancer based on analyzing the gut microbiome). All the other authors declare to have no competing interests as defined by Nature Research, or other interests that might be perceived to influence the results and/or discussion reported in this paper.

\section{References (for main text only)}

1. Ferlay, J. et al. Cancer incidence and mortality worldwide: sources, methods and major patterns in GLOBOCAN 2012. Int. J. Cancer 136, E359-86 (2015).

2. Siegel, R., Desantis, C. \& Jemal, A. Colorectal cancer statistics, 2014. CA Cancer J. Clin. 64, 104117 (2014).

3. Frank, C., Sundquist, J., Yu, H., Hemminki, A. \& Hemminki, K. Concordant and discordant familial cancer: Familial risks, proportions and population impact. Int. J. Cancer 140, 15101516 (2017).

4. Foulkes, W. D. Inherited susceptibility to common cancers. N. Engl. J. Med. 359, 2143-2153 (2008). 
5. Johnson, C. M. et al. Meta-analyses of colorectal cancer risk factors. Cancer Causes Control 24, 1207-1222 (2013).

6. Huxley, R. R. et al. The impact of dietary and lifestyle risk factors on risk of colorectal cancer: a quantitative overview of the epidemiological evidence. Int. J. Cancer 125, 171-180 (2009).

7. Schmidt, T. S. B., Raes, J. \& Bork, P. The Human Gut Microbiome: From Association to Modulation. Cell 172, 1198-1215 (2018).

8. Thomas, R. M. \& Jobin, C. The Microbiome and Cancer: Is the 'Oncobiome' Mirage Real? Trends Cancer Res. 1, 24-35 (2015).

9. Jie, Z. et al. The gut microbiome in atherosclerotic cardiovascular disease. Nat. Commun. 8, 845 (2017).

10. Pasolli, E., Truong, D. T., Malik, F., Waldron, L. \& Segata, N. Machine Learning Meta-analysis of Large Metagenomic Datasets: Tools and Biological Insights. PLoS Comput. Biol. 12, e1004977 (2016).

11. Cougnoux, A. et al. Bacterial genotoxin colibactin promotes colon tumour growth by inducing a senescence-associated secretory phenotype. Gut 63, 1932-1942 (2014).

12. Wu, S. et al. A human colonic commensal promotes colon tumorigenesis via activation of $\mathrm{T}$ helper type 17 T cell responses. Nat. Med. 15, 1016-1022 (2009).

13. Chung, L. et al. Bacteroides fragilis Toxin Coordinates a Pro-carcinogenic Inflammatory Cascade via Targeting of Colonic Epithelial Cells. Cell Host Microbe 23, 203-214.e5 (2018).

14. Kostic, A. D. et al. Genomic analysis identifies association of Fusobacterium with colorectal carcinoma. Genome Res. 22, 292-298 (2012).

15. Kostic, A. D. et al. Fusobacterium nucleatum potentiates intestinal tumorigenesis and modulates the tumor-immune microenvironment. Cell Host Microbe 14, 207-215 (2013).

16. Rubinstein, M. R. et al. Fusobacterium nucleatum promotes colorectal carcinogenesis by modulating E-cadherin/ $\beta$-catenin signaling via its FadA adhesin. Cell Host Microbe 14, 195206 (2013).

17. Yu, J. et al. Metagenomic analysis of faecal microbiome as a tool towards targeted noninvasive biomarkers for colorectal cancer. Gut 66, 70-78 (2017).

18. Feng, Q. et al. Gut microbiome development along the colorectal adenoma-carcinoma sequence. Nat. Commun. 6, 6528 (2015).

19. Zeller, G. et al. Potential of fecal microbiota for early-stage detection of colorectal cancer. Mol. Syst. Biol. 10, 766 (2014).

20. Baxter, N. T., Ruffin, M. T., Rogers, M. A. M. \& Schloss, P. D. Microbiota-based model improves the sensitivity of fecal immunochemical test for detecting colonic lesions. Genome Med. 8, 37 (2016).

21. Zackular, J. P., Rogers, M. A. M., Ruffin, M. T., 4th \& Schloss, P. D. The human gut microbiome as a screening tool for colorectal cancer. Cancer Prev. Res. 7, 1112-1121 (2014).

22. Drewes, J. L. et al. High-resolution bacterial 16S rRNA gene profile meta-analysis and biofilm status reveal common colorectal cancer consortia. NPJ Biofilms Microbiomes 3, 34 (2017).

23. Pollock, J., Glendinning, L., Wisedchanwet, T. \& Watson, M. The Madness of Microbiome: Attempting To Find Consensus 'Best Practice’ for 16S Microbiome Studies. Appl. Environ. Microbiol. 84, (2018).

24. Segata, N. On the Road to Strain-Resolved Comparative Metagenomics. mSystems 3, (2018).

25. Truong, D. T., Tett, A., Pasolli, E., Huttenhower, C. \& Segata, N. Microbial strain-level population structure and genetic diversity from metagenomes. Genome Res. 27, 626-638 (2017).

26. Pasolli, E. et al. Accessible, curated metagenomic data through ExperimentHub. Nat. Methods 14, 1023 (2017).

27. Dai, Z. et al. Multi-cohort analysis of colorectal cancer metagenome identified altered bacteria across populations and universal bacterial markers. Microbiome 6, 70 (2018).

28. Quince, C., Walker, A. W., Simpson, J. T., Loman, N. J. \& Segata, N. Shotgun metagenomics, from 
29. Wirbel, J. et al. Meta-analysis of fecal metagenomes reveals global microbial signatures that are specific for colorectal cancer. Under Submission (2018).

30. Hannigan, G. D., Duhaime, M. B., Ruffin, M. T., 4th, Koumpouras, C. C. \& Schloss, P. D. Diagnostic Potential and Interactive Dynamics of the Colorectal Cancer Virome. MBio 9, (2018).

31. Thomas, A. M. et al. Tissue-Associated Bacterial Alterations in Rectal Carcinoma Patients Revealed by 16S rRNA Community Profiling. Front. Cell. Infect. Microbiol. 6, 179 (2016).

32. Gao, Z., Guo, B., Gao, R., Zhu, Q. \& Qin, H. Microbiota disbiosis is associated with colorectal cancer. Front. Microbiol. 6, 20 (2015).

33. Ahn, J. et al. Human gut microbiome and risk for colorectal cancer. J. Natl. Cancer Inst. 105, 1907-1911 (2013).

34. Flemer, B. et al. The oral microbiota in colorectal cancer is distinctive and predictive. Gut (2017). doi:10.1136/gutjnl-2017-314814

35. Brito, I. L. et al. Mobile genes in the human microbiome are structured from global to individual scales. Nature 535, 435-439 (2016).

36. Human Microbiome Project Consortium. Structure, function and diversity of the healthy human microbiome. Nature 486, 207-214 (2012).

37. Bonder, M. J. et al. The effect of host genetics on the gut microbiome. Nat. Genet. 48, 1407 (2016).

38. Segata, N. et al. Metagenomic biomarker discovery and explanation. Genome Biol. 12, R60 (2011).

39. Xie, Y.-H. et al. Fecal Clostridium symbiosum for Noninvasive Detection of Early and Advanced Colorectal Cancer: Test and Validation Studies. EBioMedicine 25, 32-40 (2017).

40. Boleij, A., van Gelder, M. M. H. J., Swinkels, D. W. \& Tjalsma, H. Clinical Importance of Streptococcus gallolyticus infection among colorectal cancer patients: systematic review and meta-analysis. Clin. Infect. Dis. 53, 870-878 (2011).

41. Fijan, S. Microorganisms with claimed probiotic properties: an overview of recent literature. Int. J. Environ. Res. Public Health 11, 4745-4767 (2014).

42. Apweiler, R. et al. UniProt: the Universal Protein knowledgebase. Nucleic Acids Res. 32, D1159 (2004).

43. Gerner, E. W. \& Meyskens, F. L., Jr. Polyamines and cancer: old molecules, new understanding. Nat. Rev. Cancer 4, 781-792 (2004).

44. Costea, P. I. et al. Towards standards for human fecal sample processing in metagenomic studies. Nat. Biotechnol. 35, 1069-1076 (2017).

45. Riester, M. et al. Risk prediction for late-stage ovarian cancer by meta-analysis of 1525 patient samples. J. Natl. Cancer Inst. 106, (2014).

46. Kummen, M. et al. Elevated trimethylamine-N-oxide (TMAO) is associated with poor prognosis in primary sclerosing cholangitis patients with normal liver function. United European Gastroenterol J 5, 532-541 (2017).

47. Oellgaard, J., Winther, S. A., Hansen, T. S., Rossing, P. \& von Scholten, B. J. Trimethylamine Noxide (TMAO) as a New Potential Therapeutic Target for Insulin Resistance and Cancer. Curr. Pharm. Des. 23, 3699-3712 (2017).

48. Kalnins, G. et al. Structure and Function of CutC Choline Lyase from Human Microbiota Bacterium Klebsiella pneumoniae. J. Biol. Chem. 290, 21732-21740 (2015).

49. Rath, S., Heidrich, B., Pieper, D. H. \& Vital, M. Uncovering the trimethylamine-producing bacteria of the human gut microbiota. Microbiome 5, 54 (2017).

50. Pasolli, E. et al. Extensive unexplored human microbiome diversity revealed by over 150,000 genomes from metagenomes spanning age, geography, and lifestyle. Cell 176, 1-14 (2019).

51. Hothorn, T., Hornik, K., van de Wiel, M. A. \& Zeileis, A. A Lego System for Conditional Inference. Am. Stat. 60, 257-263 (2006).

52. Nielsen, H. B. et al. Identification and assembly of genomes and genetic elements in complex 


\section{Figure legends (for main text only)}

Figure 1. Reproducible taxonomic and functional microbial biomarkers across datasets when contrasting carcinoma against healthy controls (no adenoma samples considered). (A) UpSet plot showing the number of taxonomic biomarkers identified using LEfSE on MetaPhlAn2 species profiles shared by combinations of datasets (see Suppl. Table 3 for all single significant associations). (B) Pooled effect sizes for the 20 significant features with the largest effect size calculated using a meta-analysis of standardized mean differences and a random effects model on MetaPhlAn2 species abundances and on (C) HUMANn2 pathway abundances. Bold lines represent the 95\% confidence interval for the random effects model coefficient estimate . (D) Scatter plot of crude and age-, sex-, and BMI-adjusted coefficients obtained from linear models using MetaPhlAn2 species abundances. (E) Scatter plot of crude and age-, sex-, and BMI-adjusted coefficients obtained from linear models using HUMANn2 pathway abundances.

Figure 2. Assessment of prediction performances of the gut microbiome for CRC detection within and across cohorts. (A) Cross prediction matrix reporting prediction performances as AUC values obtained using a Random Forest (RF) model on species-level relative abundances (see Methods). Values on the diagonal refer to 20 times repeated 10 -fold stratified cross validations. Offdiagonal values refer to the AUC values obtained by training the classifier on the dataset of the corresponding row and applying it on the dataset of the corresponding column. The Leave-OneDataset-Out (LODO) row refers to the performances obtained by training the model on the specieslevel abundances and MetaPhlAn2 markers using all but the dataset of the corresponding column and applying it on the dataset of the corresponding column. See Extended Data 6 for the marker crossstudy validation matrix. (B) Cross prediction matrix of AUC values obtained using HUMANn2 UniRef90 gene-family abundances and HUMANn2 pathway relative abundances. See Extended Data 6 for the pathway cross-study validation matrix. (C) Prediction performances for the two Italian cohorts at increasing numbers of external datasets considered for training the model. The dark yellow line interpolates the median AUC at each number of training datasets considered. See Extended Data 7 for the plots referred to the other testing datasets. (D) Prediction performances at 
increasing number of datasets in the training, using HUMANn2 UniProt90 gene-family abundances. See Extended Data 7 for the plots referred to the other testing datasets.

Figure 3. Ranking relevance of each species in the predictive models for each dataset and identification of a minimal microbial signature for CRC detection. (A) The importance of each species for the cross-validation prediction performance in each dataset estimated using the internal RF scores. Only species appearing in the five top ranking features in at least one dataset are reported. Prediction performances at increasing number of microbial species obtained by re-training the RF classifier on the $\mathrm{N}$ top ranked features identified with a first RF model training in a cross-validation (B) and LODO-setting (C). The rankings are obtained excluding the testing dataset to avoid overfitting.

Figure 4. Choline TMA-lyase gene cutC and its genetic variants are strong biomarkers for CRCassociated stool samples. (A) Distribution of reads per kilobase million (RKPM) abundances obtained using ShortBRED for the choline TMA-lyase enzyme gene cutC. P-values were computed by two-tailed Wilcoxon Signed-Rank tests comparing values between controls and carcinomas for each dataset. (B) Forest plot reporting effect sizes calculated using a meta-analysis of standardized mean differences and a random effects model on cutC RPKM abundances between carcinomas and controls. (C) Phylogenetic tree of sample-specific cutC sequence variants identified four main sequence variants. Tips with no circles represent cutC sequence variants from genomes absent from the datasets. Taxonomy was assigned based on mapping against existing cutC sequences (criteria of $80 \%$ coverage, $>97 \%$ identity and minimum 2,000nt alignment length). (D) qPCR validation of cutC gene abundance and (E) cutC transcript abundance (normalized by total 16S rRNA gene/transcript abundance) on a subset of DNA samples from Cohort1. qPCR validation P-values are obtained by 1tail Wilcoxon Signed-Rank test.

Figure 5 - Clinical potential and validation of the predictive biomarkers. (A) Prediction performance of the taxonomic models trained on the 7 datasets of Table $\mathbf{1}$ and applied on the new validation cohorts confirmed the strong reproducibility of metagenomic models for CRC across cohorts when sufficiently large training cohorts are available. Feature ranking of the 16-species model are obtained the testing cohort to avoid overfitting. (B) Species richness, rarefied oral species richness, and cutC gene abundance (RPKM) are confirmed to be strong biomarkers of CRC in the validation datasets ${ }^{29}$. P-values underlying the panels refer to one-tailed Wilcoxon Signed Rank test; P-values overlying the panels refer to the one-sided permutation-based Wilcoxon-Mann-Whitney tests, blocked for cohort. (C) Prediction performances as AUC values on the validation cohorts when adding external set of case and controls samples from metagenomic cohorts of diseases other than CRC (Crohn's disease, ulcerative colitis, type-2 diabetes). (D) Assessment of the potential of microbiome-based prediction models in comparison and in combination with current non-invasive clinical screening tests. Models integrating our LODO machine learning approach with the FOBT or the Wif-1 Methylation tests are termed OR and AND, depending on whether only one or both need to be positive for the combined test to be positive.

\section{Tables}

Table 1. Size and characteristics of the large-scale CRC metagenomic datasets included in this study.

\begin{tabular}{|c|c|c|c|c|c|c|}
\hline Dataset & Groups (N) & $\begin{array}{c}\text { Age } \\
(\text { mean +/- s.d.) }\end{array}$ & $\begin{array}{c}\text { BMI } \\
(\text { mean +/- s.d.) }\end{array}$ & $\begin{array}{c}\text { Sex } \\
\text { F(\%)/M( } \\
\%)\end{array}$ & $\begin{array}{c}\text { Country } \\
\%\end{array}$ & $\begin{array}{c}\# \text { of reads } \\
\left(\mathrm{x} 10^{\wedge} 9\right)\end{array}$ \\
\hline
\end{tabular}




\begin{tabular}{|c|c|c|c|c|c|c|}
\hline $\begin{array}{c}\text { ZellerG_2014 } \\
\text { (Zeller et al. 2014) }\end{array}$ & $\begin{array}{l}\text { Control (61) } \\
\text { Adenoma (42) } \\
\text { CRC (53) }\end{array}$ & $\begin{array}{c}60.6+/-11.4 \\
63+/-9.1 \\
66.8+/-10.9\end{array}$ & $\begin{array}{l}24.7+/-3.2 \\
25.9+/-4.1 \\
25.5+/-5.2\end{array}$ & $\begin{array}{l}54.1 / 45.9 \\
28.5 / 71.5 \\
45.2 / 54.8\end{array}$ & France & 9.4 \\
\hline $\begin{array}{c}\text { YuJ_2015 } \\
\text { (Yu et al. 2015) }\end{array}$ & $\begin{array}{l}\text { Control (54) } \\
\text { CRC (74) } \\
\end{array}$ & $\begin{array}{l}61.8+/-5.7 \\
66+/-10.6 \\
\end{array}$ & $\begin{array}{l}23.5+/-3 \\
24+/-3.2 \\
\end{array}$ & $\begin{array}{l}38.9 / 61.1 \\
35.1 / 64.9 \\
\end{array}$ & China & 7.2 \\
\hline $\begin{array}{c}\text { FengQ_2015 } \\
\text { (Feng et al. 2015) }\end{array}$ & $\begin{array}{c}\text { Control }(61)^{*} \\
\text { Adenoma (47) } \\
\text { CRC }(46)\end{array}$ & $\begin{array}{c}67+/-6.5 \\
66.5+/-7.9 \\
67+/-10.9 \\
\end{array}$ & $\begin{array}{c}27.6+/-3.8 \\
28+/-4.7 \\
26.5+/-3.5 \\
\end{array}$ & $\begin{array}{c}41 / 59 \\
51.1 / 48.9 \\
39.1 / 60.9 \\
\end{array}$ & Austria & 8.3 \\
\hline $\begin{array}{c}\text { VogtmannE_2016 } \\
\text { (Vogtmann et al. 2016) } \\
\end{array}$ & $\begin{array}{c}\text { Control (52) } \\
\text { CRC (52) } \\
\end{array}$ & $\begin{array}{c}61.2+/-11 \\
61.8+/-13.6 \\
\end{array}$ & $\begin{array}{l}25.3+/-4.2 \\
24.9+/-4.2 \\
\end{array}$ & $\begin{array}{l}28.8 / 71.2 \\
28.8 / 71.2 \\
\end{array}$ & USA & 6.9 \\
\hline $\begin{array}{l}\text { HanniganGD_2018 } \\
\text { (Hannigan et al. 2018) }\end{array}$ & $\begin{array}{c}\text { Control (28) } \\
\text { Adenoma (27) } \\
\text { CRC (27) }\end{array}$ & NA & NA & NA & $\begin{array}{c}\text { USA (54) } \\
\text { Canada (28) }\end{array}$ & 0.5 \\
\hline $\begin{array}{l}\text { Cohort1 } \\
\text { (This study) }\end{array}$ & $\begin{array}{c}\text { Control (24) } \\
\text { Adenoma (27) } \\
\text { CRC (29) }\end{array}$ & $\begin{array}{l}67.9+/-7.1 \\
62.8+/-8.6 \\
71.4+/-8.2 \\
\end{array}$ & $\begin{array}{l}25.3+/-3.5 \\
25.3+/-4.1 \\
25.7+/-4.1 \\
\end{array}$ & $\begin{array}{l}45.8 / 54.1 \\
40.7 / 59.3 \\
20.7 / 79.3 \\
\end{array}$ & Italy & 8.2 \\
\hline $\begin{array}{c}\text { Cohort2 } \\
\text { (This study) }\end{array}$ & $\begin{array}{l}\text { Control (28) } \\
\text { CRC (32) } \\
\end{array}$ & $\begin{array}{r}57.8+/-8.3 \\
58.4+/-8.4 \\
\end{array}$ & $\begin{array}{l}24.6+/-3.8 \\
26.8+/-4.3 \\
\end{array}$ & $\begin{array}{l}42.9 / 57.1 \\
28.1 / 71.9 \\
\end{array}$ & Italy & 5.1 \\
\hline Total & $\begin{array}{c}\text { Control (308) } \\
\text { Adenoma (143) } \\
\text { CRC (313) }\end{array}$ & -- & -- & -- & -- & 45.6 \\
\hline
\end{tabular}

${ }^{*}$ Numbers differed from the original sample numbers $(\mathrm{N}=61$ instead of 63$)$ reported in the article due to metadata and/or sequence processing issues. $\mathrm{NA}=$ Not available.

\section{Methods}

\section{Italian cohorts of CRC patients, adenomas and controls}

The two clinical studies performed here were approved by the relevant ethics committees (Cohort1: Ethics committee of Azienda Ospedaliera "SS. Antonio e Biagio e C. Arrigo" of Alessandria, Italy, protocol N. Colorectal_miRNA_CEC2014 and Cohort2: Ethics committee of European Institute of Oncology of Milan, Italy, protocol N. R107/14-IEO 118) and informed consent was obtained from all participants.

For Cohort1, samples were collected from patients at the Clinica S. Rita in Vercelli, Italy. Patients with hereditary CRC syndromes, with previous history of CRC, and with uncompleted or poorly cleaned colonoscopy, were excluded from the study. Patients were recruited at initial diagnosis and had not received any treatment prior to fecal sample collection. Subjects reporting the use of antibiotics during the 6 months prior to the sample collection were excluded from the study. On the basis of colonoscopy results, recruited subjects were classified into three categories: 1 ) healthy subjects: individuals with colonoscopy negative for tumor, adenomas and other diseases; 2) adenoma patients: individuals with colorectal adenoma/s; and 3) CRC patients: individuals with newly diagnosed CRC. A total of 93 subjects were initially recruited, and the 80 that passed quality control (see below) are divided into 29 CRC patients, 27 adenomas and 24 controls. An additional 13 subjects that presented inflammatory GI tract diseases (ulcerative and Crohn's colitis, diverticular diseases) were recruited and fecal samples were subsequently used as a part of the final validation. Stool was collected in Stool Nucleic Acid Collection and Transport Tubes with RNA stabilising solution (Norgen Biotek Corp) and returned before performing the colonoscopy. Aliquots of the stool samples were stored at $-80^{\circ} \mathrm{C}$ until use. DNA was extracted from aliquot of fecal samples using the Qiamp DNA stool kit (Qiagen) following manufacturer's instructions. Total RNA from faeces was extracted using the Stool Total RNA Purification Kit (Norgen Biotek Corp) following manufacturer's instructions. 
For Cohort2, a total of 60 subjects were recruited at the European Oncology Institute in Milan, Italy and were divided into $32 \mathrm{CRC}$ patients and 28 controls. Controls, matched for age ( \pm 5 years) and season of blood withdrawn ( \pm 2 years), were recruited among subjects who underwent recent colonoscopy and had negative or no other relevant gastrointestinal disorders. Subjects reporting the use of antibiotics in the 6 months prior to the sample collection were excluded. Fecal samples were collected from healthy subjects and patients (before surgery, or any other cancer treatment) and directly frozen at $-80^{\circ} \mathrm{C}$ in resuspension buffer (TES buffer: $50 \mathrm{mM}$ Tris-HCL, $10 \mathrm{mM} \mathrm{NaCl}, 10 \mathrm{mM}$ EDTA, pH 7.5) and kept in liquid nitrogen until DNA extraction. DNA was extracted from fecal samples with the GNOME DNA isolation kit (MP).

747 Sequencing libraries were prepared using the NexteraXT DNA Library Preparation Kit (Illumina, 748 California, USA), following the manufacturer's guidelines. Sequencing was performed on the HiSeq2500 (Illumina, California, USA) at the internal sequencing facility of the Centre for Integrative Biology, Trento, Italy.

\section{Public metagenomic cohorts of CRC patients, adenomas and controls.}

We downloaded 5 public fecal shotgun CRC datasets covering samples from 6 different countries, totaling 313 CRC patients, 143 adenomas and 308 controls (Table 1) and now available in curatedMetagenomicData ${ }^{26}$. We manually curated metadata tables for the public cohorts according to the curatedMetagenomicData ${ }^{26} \mathrm{R}$-package grammatical rules. The metadata table includes ten fields (sampleID, subjectID, body_site, country, sequencing_platform, PMID, number_reads, number_bases, minimum_read_length, median_read_length) that are mandatory for all datasets in addition to other fields that are dataset-specific.

\section{Description of the two validation cohorts}

We consider an additional set of samples from two independent cohorts that were not available at the time we performed the meta-analysis on the other seven datasets, and we thus used them as validation cohorts. Validation Cohort1 consists of 60 CRC metagenomes collected in Germany after colonoscopy and 65 sex and age-matched healthy controls and is described in depth in the study accompanying this work ${ }^{29}$. Shotgun metagenomic sequencing was performed by Illumina HiSeq 2000 / 2500 / 4000 (Illumina, San Diego, USA) platforms at the Genomics Core Facility, European Molecular Biology Laboratory, Heidelberg. Validation Cohort2 consists of $40 \mathrm{CRC}$ samples and 40 controls from a Japanese cohort from Tokyo. DNA was extracted for Validation Cohort2 from frozen fecal samples by bead-beating using the GNOME DNA Isolation Kit (MP Biomedicals, Santa Ana, CA) and DNA quality was assessed with an Agilent 4200 TapeStation (Agilent Technologies, Santa Clara CA). Sequencing libraries were generated with a Nextera XT DNA Sample Prep Kit (Illumina, San Diego, CA) and shotgun metagenomics of fecal samples was carried out on the HiSeq2500 platform (Illumina) at a targeted depth of $5.0 \mathrm{~Gb}$ (150-bp paired end reads).

The samples and clinical information used from both validation cohorts in this study were obtained under conditions of informed consent and with approval of the institutional review boards of each participating institute.

\section{Public metagenomic cohorts of non-CRC patients.}

We used the curatedMetagenomicData ${ }^{26}$ resource to retrieve taxonomical and functional potential profiles as well as metadata of three public cohorts: NielsenHB_2014 ${ }^{52}$ comprising 21 Crohn Disease 
174 controls; and we downloaded 1339 metagenomes from the Human Microbiome Consortium phase- 2 cohort 55 , comprising 598 Crohn Disease patients, 375 Ulcerative Colitis patients and 365 controls.

\section{Sequence pre-processing, taxonomic and functional profiling}

Fecal metagenomic shotgun sequences obtained from the Italian cohorts were subjected to a preprocessing pipeline whereby sequences were quality filtered using trim_galore (parameters: -nextera --stringency 5 --length 75 --quality 20 --max_n 2 --trim-n) discarding all reads with quality less than 20 and shorter than 75 nucleotides. Filtered reads were then aligned to the human genome (hg19) and the PhiX genome for human and contaminant DNA removal using bowtie2 ${ }^{61}$. Thirteen samples, having less than $2 \mathrm{~Gb}$ of host-decontaminated DNA, were excluded from the study.

We used MetaPhlAn2 ${ }^{62}$ for quantitative profiling the taxonomic composition of the microbial communities of all metagenomic samples, whereas HUMANn2 ${ }^{63}$ was used to profile pathway and gene family abundances. The profiles generated for the 6 public cohorts, along with their metadata, and the two newly sequenced cohorts are available through the curatedMetagenomicData R package 26. Oral species were defined in this work by analyzing the 463 oral samples from the Human Microbiome Project dataset ${ }^{36}$ and the 140 saliva samples from ${ }^{35}$. Specifically, all species with $>0.1 \%$ abundance and $>5 \%$ prevalence were deemed to be of oral origin. For F. nucleatum marker analysis, we extracted MetaPhlAn2 clade-specific markers from each sample sam file and considered a marker to be present if the coverage was greater than zero.

\section{The Random Forest based machine learning approach}

Our machine learning analyses exploited 4 types of microbiome quantitative profiles: taxonomic species-level relative abundances and marker presence or absence patterns inferred by MetaPhlAn2 62 , gene-family and pathway relative abundances estimated by HUMAnN2 ${ }^{63}$.

All machine learning experiments used Random Forest ${ }^{64}$, as this algorithm has been shown to outperform, on average, other learning tools for microbiome data ${ }^{10}$. The code generating the analyses and the figures is available at https://bitbucket.org/CibioCM/multidataset machinelearning/src/, and is based on MetAML ${ }^{10}$ with the Random Forest implementation taken from Scikit-Learn version 0.19.0, 65. We used an ensemble of 1000 estimator trees and Shannon entropy to evaluate the quality of a split at each node of a tree. The two hyper-parameters for the minimum number of samples per leaf and for the number of features per tree are set as indicated elsewhere 66 to 5 and 30\% respectively. For the marker presence/absence profiles we used a number of features equal to the square root of the total number of features, and this percentage was further decreased to $1 \%$ when using gene-family profiles as they have a substantially higher number of features $(>2 \mathrm{M})$. The experiments ran on reduced sets of input features (Figure 4, Suppl. Fig. 19) avoided feature subsampling when less than 128 features were used (Suppl. Fig. 19).

\section{Application and evaluation of the learning models}

The inside-dataset prediction capability was measured through 10-fold cross-validation, stratified so each fold contained a balanced proportion of positive and negative cases. The procedure of forming the folds and assessing the models was repeated 20 times. The final result is therefore an average over 200 validation folds. In the cross-study validation, datasets are considered two by two: one is used for training the model, the other to validate. 
The Leave-one-dataset-out (LODO) approach consists of training the model on the pooled samples from all cohorts except the one used for model testing. This mimics the scenario in which all the available samples from multiple cohorts are used to predict CRC-positive samples in a newly established cohort. As a part of the meta-analysis, we iterated along all the cohorts, performing a LODO validation on each set of samples (Figure 2).

\section{Additional validation experiments on independent datasets and other diseases}

We built a validation LODO model trained on MetaPhlAn2 taxonomic abundances from the previously described set of 7 cohorts and applied it to the independent validation cohorts. To test the performance of the model when challenged with other diseases, we selected 4 metagenomic cohorts 52-55 covering 3 non-CRC diseases (ulcerative colitis - UC, Crohn's disease - CD, and type-2 diabetes T2D) and we used them for further experiments. For each disease (UC, CD, T2D) in each dataset, we randomly drawn 60 samples from the control class as well as 60 samples from the cases and added them to each validation dataset in turn, labelled as controls. The random selection was repeated ten times, and the validation AUC computed on the model's prediction accordingly. The rationale is to observe the decrease in AUC when the external cases are added to the controls of the validation cohort with respect the addition of healthy controls.

Specificity of the prediction model was also assessed by the addition of 13 IBD samples to Cohort1: we used the 13 samples either as controls for Cohort1 or added to the original controls; we performed a cross-validation and a LODO on Cohort1 (no validation cohorts in the training) using MetaPhIAn2 microbial species.

To assess the prediction ability of our Random Forest approach with respect to more traditional noninvasive tests like the FOBT and the Wif-1 Methylation test, we recorded the true positive rate (sensitivity) and the false positive rate (1 - specificity) for a subset of the ZellerG_2014 cohort according to these two tests and one-hundred positive detection thresholds in the case of Random Forest models. We then combined the Random Forest approach with the two tests in turn, first assigning the positive class when both predictors are positive ("AND" model) secondly when just one predictor is ("OR" model).

\section{Statistical analysis}

Univariate analyses on a per dataset basis was performed using LEfSe 38 to identify features that were statistically different among groups and estimate their effect size. ANCOM was also applied ${ }^{67}$ but showed reduced power on our datasets (e.g. it identified $F$. nucleatum as a biomarker in only one dataset) probably due to the low relative abundance of CRC biomarkers that are thus only minimally affected by the problem of compositionality. For these reasons, we chose to use LEfSe for the univariate analysis and focused on the biomarkers with the highest effect size. To overcome the limitations of univariate statistics, we performed multivariate analysis using linear models fitted to the data using the limma R package 68 and possible confounders such as age, sex and BMI were included in the models. For the meta-analysis on taxonomic and functional profiles, we converted relative abundances to arcsine-square root transformed proportions and used the escalc function from the R metafor package that employed Cohen's standardized mean difference statistic to calculate random effects model estimates. We quantified study heterogeneity using the $\mathrm{I}^{2}$ estimate (percentage of variation reflecting true heterogeneity) as well as Cochran's $Q$ test to assess statistically significant heterogeneity. P-values obtained from the random effects models were corrected for multiple hypothesis testing correction using the Benjamini-Hochberg procedure and corrected $P<0.05$ were considered statistically significant. Cluster analysis was conducted by 
calculating distance matrices from phylogenetic trees using the APE R-package, clustering using partitioning around medoids (PAM) and computing clusters' prediction strength using the cluster Rpackage. When validating differential species richness, oral-species richness, and increased abundance of the cutC gene, we also assessed significance through one-sided permutation-based Wilcoxon-Mann-Whitney tests where we blocked for cohort ${ }^{51}$, as implemented in the 'coin' R75 th percentiles. The upper and lower whiskers extend from the hinges to the largest (or smallest) value no further than $1.5 *$ inter-quartile range (IQR) from the hinge, defined as the distance between the 25th and 75th percentiles. Data beyond the end of the whiskers are plotted individually.

\section{Identification and quantification of the genes encoding TMA producing enzymes}

877 In order to obtain a more comprehensive database of choline TMA-lyase enzyme sequences, we downloaded amino acid sequences that matched the keywords "cutC" and "cutD" from UniProt90 42, mapped their IDs to EMBL CDS using UniParc and used the resulting DNA sequences to search, using BLASTn 69 , all 48,902 Prokka ${ }^{70}$ annotated genomes available in our repository ${ }^{71}$. Matching queries were filtered to include only alignments with $>80 \%$ identity and length $>1000 \mathrm{nt}$ for $\mathrm{cutC}$ and $>800 \mathrm{nt}$ for $c u t D$, and an e-value $<1 \mathrm{e}-15$. We used ShortBRED 72 to identify short seed sequences that were representative of the filtered queries using UniProt's UniRef100 database and quantified them in the metagenomes, normalizing by the number of reads per kilobase million (RPKM). The pipeline was also applied to identify and quantify the L-carnitine/gamma-butyrobetaine antiporter (caiT) and the dioxygenase yea $\mathrm{W}$, responsible for producing TMA preferentially via carnitine degradation. In order to investigate differences in cutC sequence types, we clustered cutC sequences at $97 \%$ sequence identity using UCLUST ${ }^{73}$ and aligned raw reads to the clustered cutC database using bowtie $2{ }^{61}$. From the bam files we calculated the breadth and depth of each sequence and generated their corresponding consensus sequence using Samtools ${ }^{74}$ and VCF utils 75 . We chose the representative cutC sequence for each sample as the one with the highest breadth or the highest depth, if there were multiple cutC sequences with the same breadth. We filtered representative cutC sequences from each sample to include only those with a breadth $>80 \%$, aligned them using MAFFT ${ }^{76}$, built a phylogenetic tree using fastTree 77 which was refined using RAxML ${ }^{78}$ and visualized using GraPhlAn 79.

\section{Validation of cutC gene and transcript abundances by qPCR}

Real time qPCR was used to assess differences in cutC genes and transcripts between CRC samples and controls. We used a previously described protocol ${ }^{49}$ which employs $16 \mathrm{~S}$ rRNA abundances as an internal sample normalization. For first strand cDNA synthesis, $400 \mathrm{ng}$ of RNA templates were retrotranscribed using the High-capacity cDNA Reverse Transcription Kits with Random Primers (Thermofisher Scientific) following the manufacturer's instructions. The cutC and 16S rRNA genes (and transcripts from cDNA) were amplified using degenerate primers and cycling conditions as described previously ${ }^{49}$. Briefly, reactions were performed in triplicate with $10 \mathrm{ng}$ of template DNA or $30 \mathrm{ng}$ of cDNA on the Rotor Gene Q (QIAGEN) using HOT FIREPol EvaGreen qPCR mix (SOLIS BIODYNE) with a final primer concentration of 0.5 a M (16S) or 0.75 a $\mathrm{M}$ (cutC). Cycling conditions were as follows: initial denaturation of $95^{\circ} \mathrm{C}$ for $15 \mathrm{~min}$; followed by 40 cycles of denaturing at $95^{\circ} \mathrm{C}$ for $45 \mathrm{~s}$, annealing at $57^{\circ} \mathrm{C}(\mathrm{cutC})$ or $55^{\circ} \mathrm{C}$ for (16S) for $45 \mathrm{~s}$ and an extension step of $72^{\circ} \mathrm{C}$ for $45 \mathrm{~s}$. Melting curves were subsequently performed for all reactions using the following program: $95^{\circ}$ for 5 $\mathrm{s}$, followed by $65^{\circ} \mathrm{C}$ for $60 \mathrm{~s}$, and a final continuous reading step of seven acquisitions per second between 65 and $97^{\circ} \mathrm{C}$. 
Quantification of the $\operatorname{cutC}$ gene by means of qPCR protocol was applied to 44 samples belonging to Cohort1 for which enough DNA was available. Samples for which either the cutC or the 16S rRNA amplification failed were removed and we retained measurements for a total of 16 CRC and 19 control samples. Relative gene fold change was calculated by applying the $\Delta \Delta \mathrm{Ct}$ method ${ }^{80}$, with $\Delta \mathrm{Ct}$ calculated as difference between cutC and 16S rRNA Ct values. Significance of the cutC vs. 16S rRNA comparison was assessed through the one-tailed Wilcoxon Signed Rank test. The same procedure was applied on the quantification of cutC and 16S rRNA transcripts from cDNA, which was computed using $26 \mathrm{CRC}$ and 20 control samples for which we obtained a reliable quantification of both cutC and 918 16S rRNA.

\section{Data Availability}

920 Nucleotide sequences for the two new Italian cohorts are available in the Sequence Read Archive (SRA) under the accession number SRP136711. MetaPhlAn2 and HUMANn2 profiles for the new cohorts were also added to the curatedMetagenomicData $\mathrm{R}$ package along with their corresponding metadata. Validation Cohort1 is available in the European Nucleotide Archive (ENA) under the study identifier PRJEB27928, Validation Cohort2 is available in the DDBJ databases under the accession number DRA006684.

61. Langmead, B. \& Salzberg, S. L. Fast gapped-read alignment with Bowtie 2. Nat. Methods 9, 357 (2012).

62. Truong, D. T. et al. MetaPhlAn2 for enhanced metagenomic taxonomic profiling. Nat. Methods 12, 902-903 (2015).

63. Abubucker, S. et al. Metabolic reconstruction for metagenomic data and its application to the human microbiome. PLoS Comput. Biol. 8, e1002358 (2012).

64. Breiman, L. Random Forests. Mach. Learn. 45, 5-32 (2001).

65. Pedregosa, F. et al. Scikit-learn: Machine Learning in Python. J. Mach. Learn. Res. 12, 28252830 (2011).

66. Hastie, T., Tibshirani, R. \& Friedman, J. The Elements of Statistical Learning. 1, (SpringerVerlag New York, 2009).

67. Mandal, S. et al. Analysis of composition of microbiomes: a novel method for studying microbial composition. Microb. Ecol. Health Dis. 26, 27663 (2015).

68. Ritchie, M. E. et al. limma powers differential expression analyses for RNA-sequencing and microarray studies. Nucleic Acids Res. 43, e47 (2015).

69. Altschul, S. F., Gish, W., Miller, W., Myers, E. W. \& Lipman, D. J. Basic local alignment search tool. J. Mol. Biol. 215, 403-410 (1990).

70. Seemann, T. Prokka: rapid prokaryotic genome annotation. Bioinformatics 30, 2068-2069 (2014).

71. Segata, N., Börnigen, D., Morgan, X. C. \& Huttenhower, C. PhyloPhlAn is a new method for improved phylogenetic and taxonomic placement of microbes. Nat. Commun. 4, 2304 (2013).

72. Kaminski, J. et al. High-Specificity Targeted Functional Profiling in Microbial Communities with ShortBRED. PLoS Comput. Biol. 11, e1004557 (2015).

73. Edgar, R. C. Search and clustering orders of magnitude faster than BLAST. Bioinformatics 26, 2460-2461 (2010).

74. Li, H. et al. The Sequence Alignment/Map format and SAMtools. Bioinformatics 25, 2078-2079 
956 75. Danecek, P. et al. The variant call format and VCFtools. Bioinformatics 27, 2156-2158 (2011).

957 76. Katoh, K. \& Standley, D. M. MAFFT multiple sequence alignment software version 7:

958 improvements in performance and usability. Mol. Biol. Evol. 30, 772-780 (2013).

959 77. Price, M. N., Dehal, P. S. \& Arkin, A. P. FastTree 2--approximately maximum-likelihood trees

960 for large alignments. PLoS One 5, e9490 (2010).

961 78. Stamatakis, A. RAxML version 8: a tool for phylogenetic analysis and post-analysis of large

962 phylogenies. Bioinformatics 30,1312-1313 (2014).

963

964

79. Asnicar, F., Weingart, G., Tickle, T. L., Huttenhower, C. \& Segata, N. Compact graphical

965 representation of phylogenetic data and metadata with GraPhlAn. PeerJ 3, e1029 (2015).

966

80. Livak, K. J. \& Schmittgen, T. D. Analysis of relative gene expression data using real-time quantitative PCR and the 2(-Delta Delta C(T)) Method. Methods 25, 402-408 (2001). 


\section{$A$}

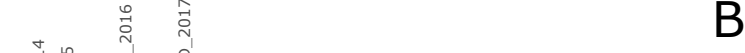

Intersection Size

耎 0 10

20

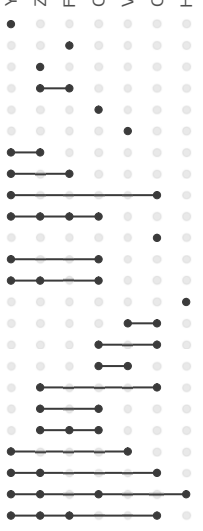

0

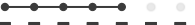

$\stackrel{N}{N} 20$

in

ث 40


30 40

B

\begin{tabular}{|ll|}
\hline & Random Effects \\
$\triangle$ & Cohort1 \\
$\bigcirc$ & HanniganGD_2017 \\
$\square$ & VogtmannE_2016 \\
$*$ & FengQ_2015 \\
$\times$ & YuJ_2015 \\
+ & ZellerG_2014 \\
\hline
\end{tabular}

Fusobacterium nucleatum

Parvimonas micra

Parvimonas spp.

Gemella morbillorum

Peptostreptococcus stomatis

Solobacterium moorei

Clostridium symbiosum

Anaerococcus vaginalis

Porphyromonas asaccharolytica

Prevotella intermedia

Bacteroides fragilis

Porphyromonas somerae

Anaerococcus obesiensis

Porphyromonas uenonis

Peptostreptococcus anaerobius

Streptococcus constellatus

Granulicatella adiacens
Carcinoma Enriched



○ $\triangle$ 다 *

Q $\triangle * *$

Q) $\boxminus \bullet$ $\Delta * \triangle$

* $+x$

$\times \infty 1 *$

$\square \times x$

O

$\Delta x$

$\infty \bullet 4 *$

$\triangle$

$\times$ *

$\longrightarrow$

$\triangle \sim$

* $\diamond$

\section{C}

L-lysine fermentation to acetate and butanoate

L-glutamate degradation VIII

$\mathrm{L}$-alanine fermentation to propanoate and acetate

gluconeogenesis I

L-histidine degradation I

CMP-3-deoxy-D-manno-octulosonate biosynthesis !

menaquinol-8 biosynthesis II

L-tryptophan biosynthesis
Roseburia intestinalis

Gordonibacter pamelaeae

Bifidobacterium catenulatum 0.5
1.0
E

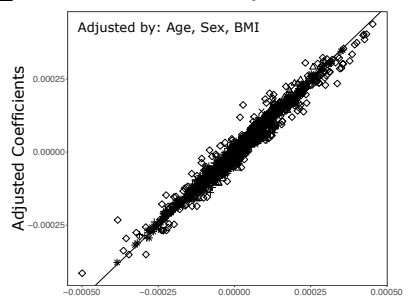

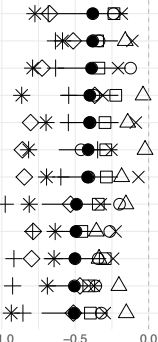

Crude Coefficients

Crude Coefficients

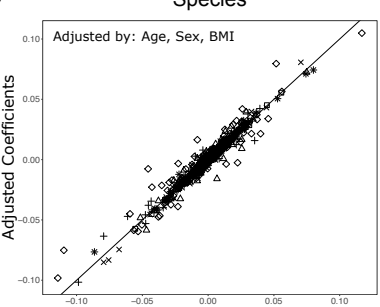

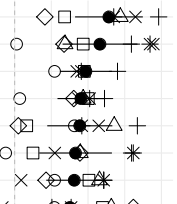

$\triangle \quad$ Calvin-Benson-Bassham cycle thiamin salvage pentose phosphate pathway

L-serine and glycine biosynthesis I chorismate biosynthesis I

pyruvate fermentation to isobutanol aromatic amino acid biosynthesis galactose degradation I starch degradation $\mathrm{V}$

S-adenosyl-L-methionine cycle I stachyose degradation

D-galactose degradation V 
$A$

Random Forest Feature Ranking

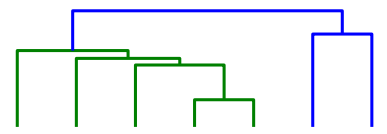

Peptostreptococcus stomatis

Fusobacterium nucleatum

Parvimonas spp.

\begin{tabular}{|c|c|c|c|c|c|}
\hline 31 & 18 & 4 & 1 & 1 & 177221 \\
\hline 1 & 2 & 114 & 4 & 11 & 346 \\
\hline 26 & 4 & 1 & 3 & 2 & 300207 \\
\hline
\end{tabular}

Porphyromonas asaccharolytica

Gemella morbillorum

$\begin{array}{lllllll}3 & 5 & 213 & 59 & 70 & 20 & 99\end{array}$

$\begin{array}{llllllllll}\text { Clostridium symbiosum } & 6 & 105 & 133 & 22 & 4 & 8 & 205\end{array}$

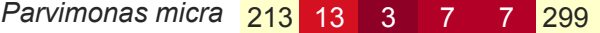

$\begin{array}{lllllllll}\text { Escherichia coli } & 2 & 33 & 20 & 44 & 73 & 2 & 58\end{array}$

\begin{tabular}{ll|l|l|l|l|l|l|} 
Streptococcus parasanguinis & 29 & 21 & 80 & 38 & 100 & 95 & 4
\end{tabular}

$\begin{array}{lllllllll}\text { Clostridium leptum } & 5 & 111 & 40 & 88 & 108 & 67 & 59\end{array}$

\begin{tabular}{lllll|lllll} 
Clostridium hathewayi & 67 & 57 & 122 & 6 & 5 & 24 & 202
\end{tabular}

Anaerotruncus colihominis $40 \begin{array}{llllllll}40 & 137 & 97 & 90 & 5 & 223\end{array}$

$\begin{array}{lllllllll}\text { Prevotella copri } & 21 & 1 & 55 & 140 & 52 & 65 & 158\end{array}$ Lachnospiraceae 31 57FAA CT1 $44 \begin{array}{llllllll}75 & 123 & 110 & 130 & 14 & 69\end{array}$

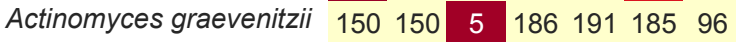

Alistipes spp. $2 2 5 2 0 7 2 7 6 2 1 8 2 3 8 1 2 8 \longdiv { 2 }$

Lachnospiraceae 81 57FAA $193 \quad 65159195234129 \quad 3$



\begin{tabular}{l|l|l|llllll} 
Ruminococcus gnavus & 16 & 71 & 107 & 76 & 84 & 93 & 5
\end{tabular}

\begin{tabular}{lll|l|l|l|l|l|} 
Bifidobacterium longum & 103 & 96 & 52 & 45 & 35 & 4 & 36
\end{tabular}

$\begin{array}{lllllllllll}\text { Subdoligranulum spp. } & 46 & 81 & 36 & 46 & 76 & 3 & 6\end{array}$



$\begin{array}{lllllllll}\text { Eubacterium eligens } & 63 & 86 & 70 & 11 & 15 & 1 & 70\end{array}$

$\begin{array}{llllllllll}\text { Streptococcus salivarius } & 25 & 28 & 54 & 2 & 66 & 23 & 1\end{array}$
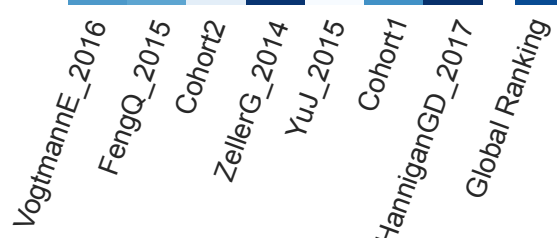

FengQ_2015 ZellerG_2014 Cohort2 • HanniganGD_2017

YuJ_2015 VogtmannE_2016 Cohort1 • Cross-Validation
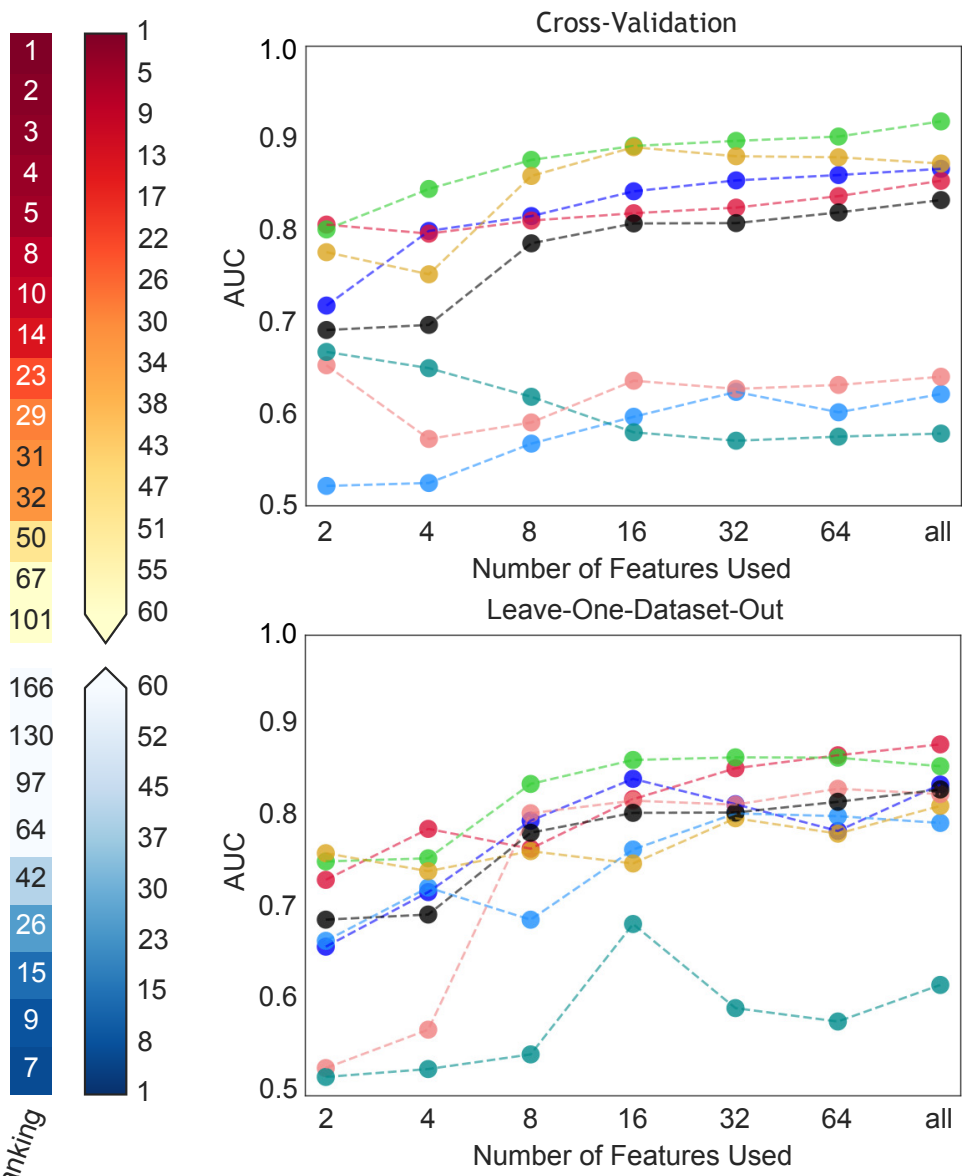

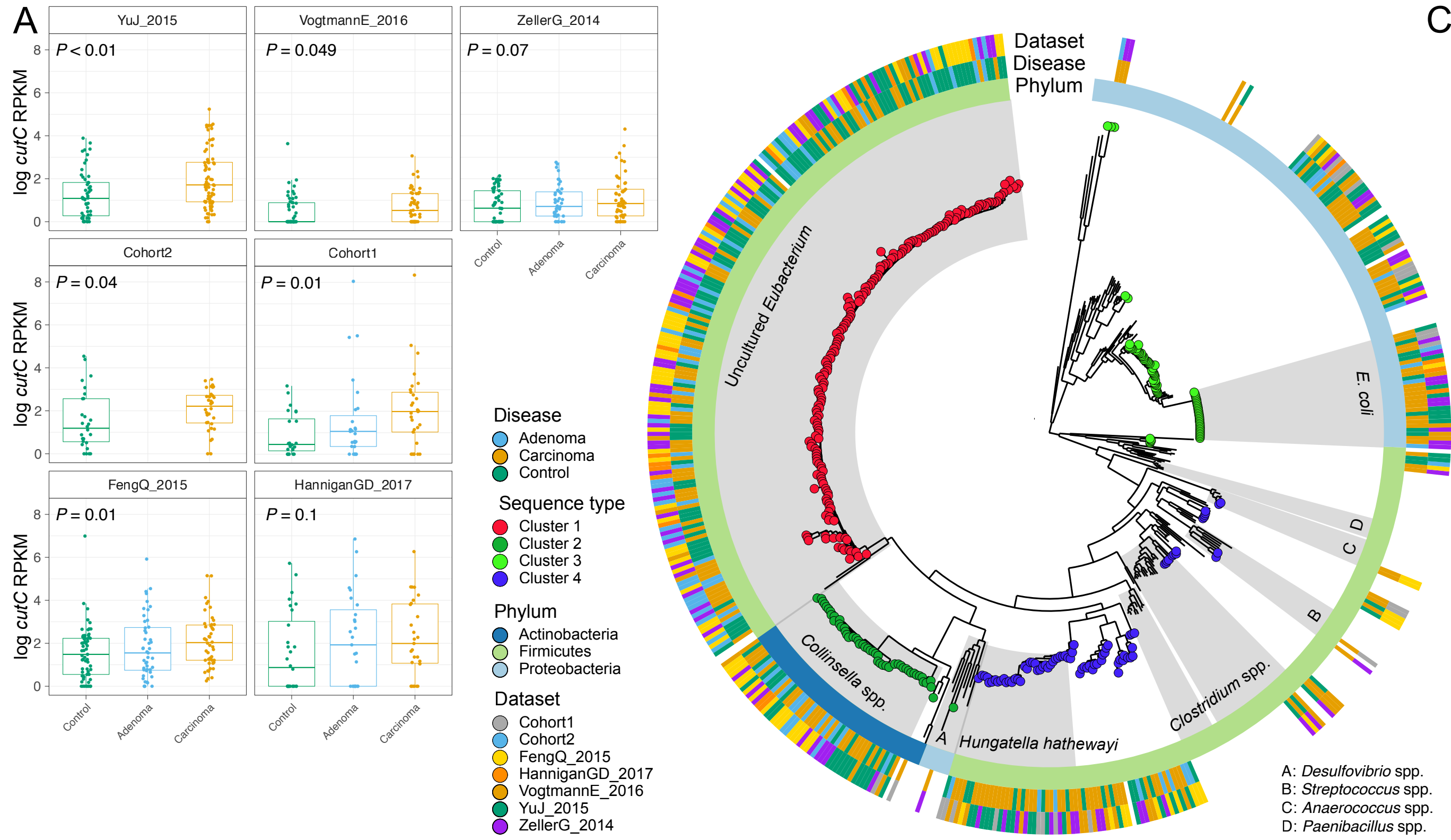

B

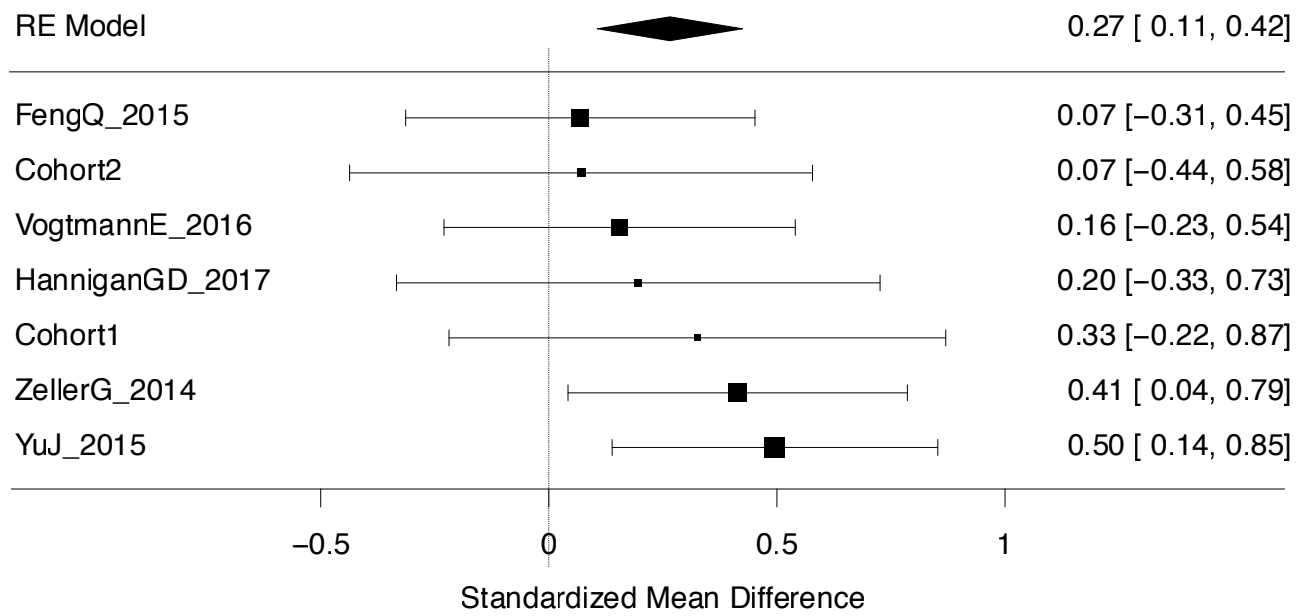

D

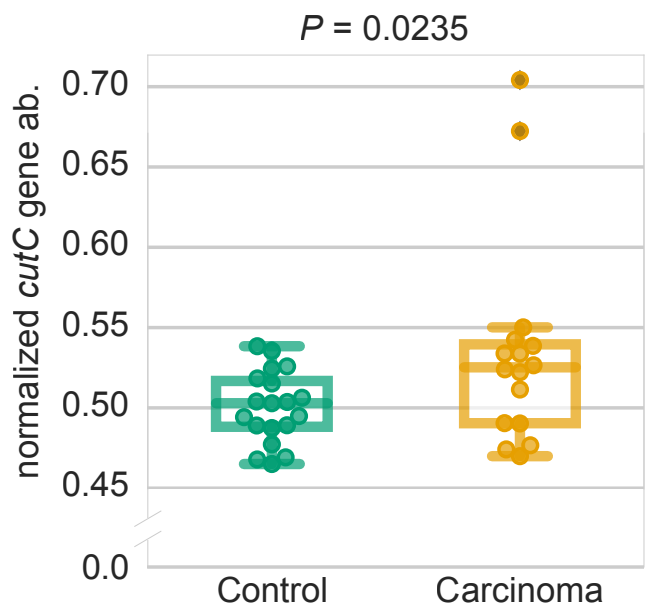

E

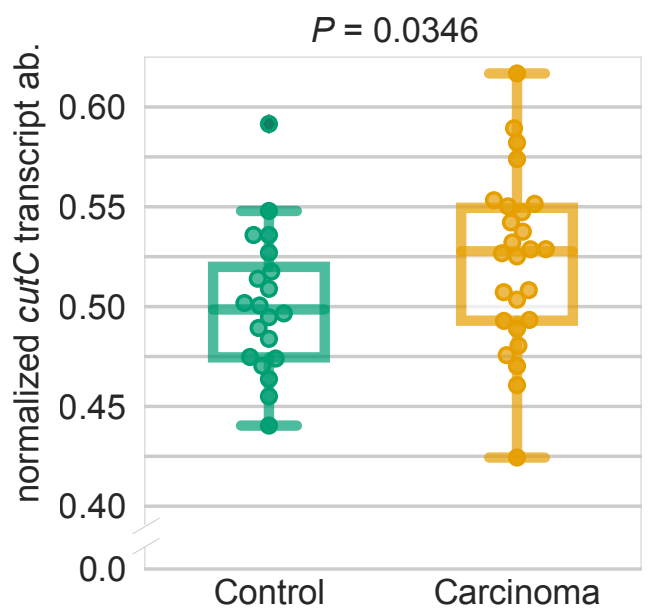


A Random Forest Model

\begin{tabular}{l|l|l|l|} 
V. Cohort 1 & 0.90 & 0.91 & 1.0 \\
\hline V. Cohort 2 & 0.81 & 0.77 & 0.5 \\
\hline
\end{tabular}



B Validation of predictive biomarkers

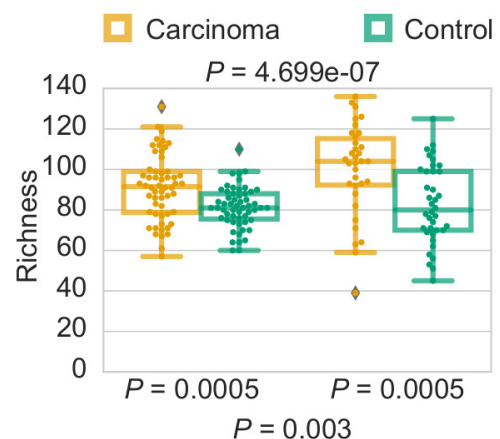

0
0
0
0
0
0
0
0
0
0
0
0
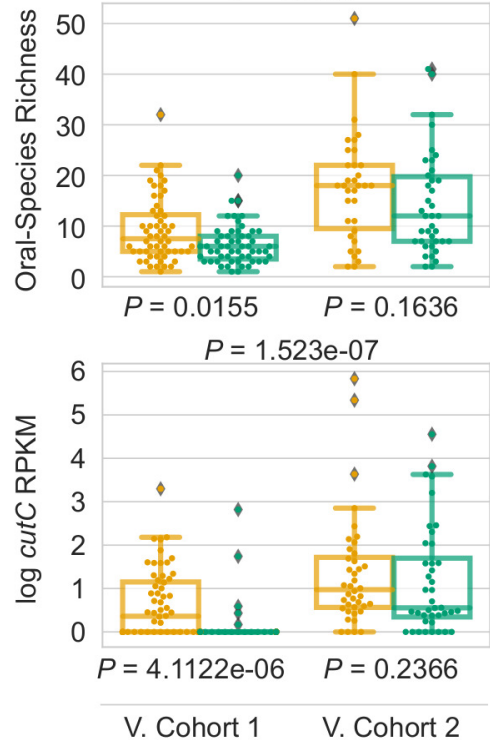

CRC-specificity of the predictive models

C Validation Cohort 1
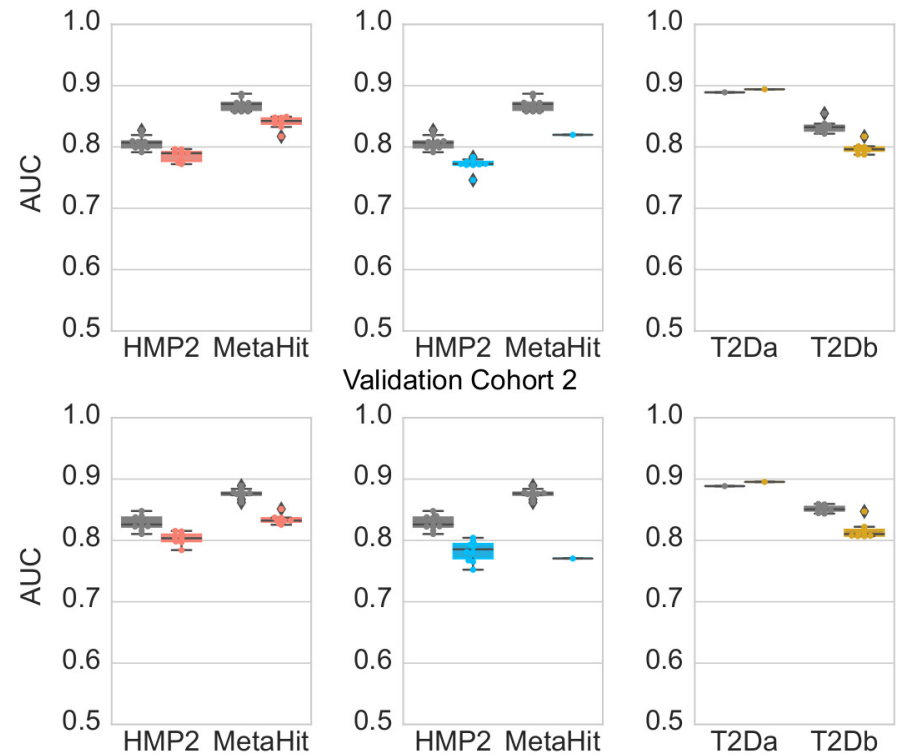

\section{Validation Cohort 2}
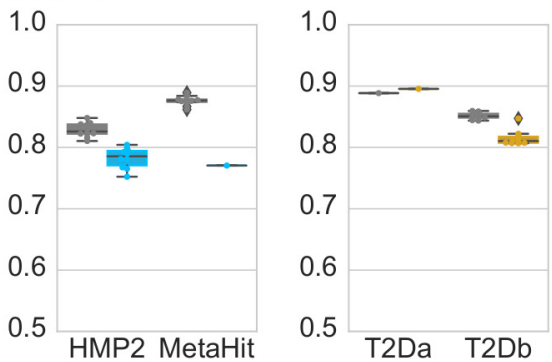

Control

Crohn's Disease

Ulcerative Colitis

Type-2 Diabetes

Relationship to other non-invasive tests

- LODO

- $\mathrm{LODO}+\mathrm{FOBT}$ "AND" $=\mathrm{LODO}+\mathrm{FOBT}$ "OR"

- LODO + WIF-1 "AND" — LODO + WIF-1 "OR"

- FOBT

- WIF-1 Methylation

All Features

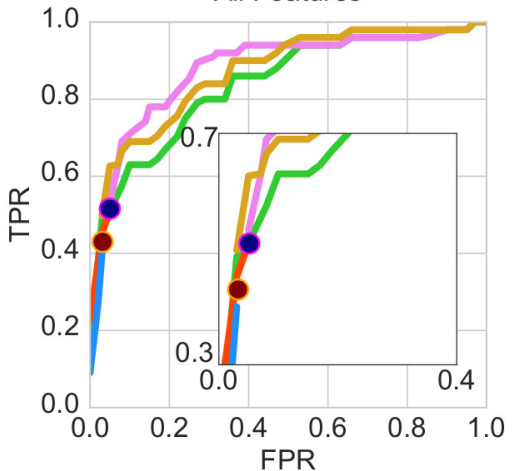

16 Features

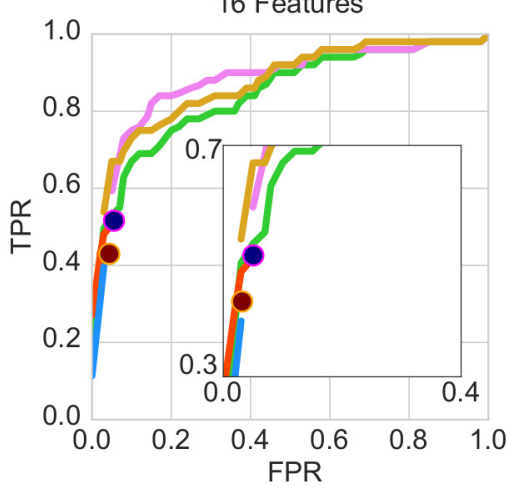

